\title{
Magnetic properties of small Pt-capped Fe, Co, and Ni clusters: A density functional theory study
}

\author{
Sanjubala Sahoo, * Alfred Hucht, Markus E. Gruner, Georg Rollmann, and Peter Entel \\ Faculty of Physics and Center for Nanointegration CeNIDE, University of Duisburg-Essen, Lotharstr. 1, D-47048 Duisburg, Germany
}

Andrei Postnikov

LPMD, Paul Verlaine University-Metz, 1 Boulevard Arago, F-57078 Metz, France

\author{
Jaime Ferrer and Lucas Fernández-Seivane \\ Departamento de Física, Universidad de Oviedo, 33007 Oviedo, Spain \\ Manuel Richter and Daniel Fritsch \\ IFW Dresden e.V., P.O. Box 270116, D-01171 Dresden, Germany
}

\author{
Shreekantha Sil \\ Department of Physics, Visva Bharati University, Santiniketan, 731235 West Bengal, India \\ (Received 21 May 2010; revised manuscript received 15 July 2010; published 13 August 2010)
}

\begin{abstract}
We have undertaken theoretical studies of spin and orbital magnetic moments as well as magnetic anisotropy energies for $M_{13}(M=\mathrm{Fe}, \mathrm{Co}, \mathrm{Ni})$ and $M_{13} \mathrm{Pt}_{n}(n=3,4,5,20)$ clusters including the spin-orbit coupling in the framework of density functional theory. For all $M_{13}$ clusters considered we find tendencies for small structural distortions which can be characterized by either Jahn-Teller (JT) or Mackay transformations (MT). The magnetic anisotropy energy (MAE) along with the spin and orbital moments are calculated for $M_{13}$ icosahedral clusters and the angle-dependent energy differences are modeled using a Néel model with local anisotropies. From our studies, the MAE for JT-distorted $M_{13}$ clusters are found to be larger relative to the MT clusters and more than two orders of magnitude larger compared to the corresponding bec or fcc bulk values. In addition, we demonstrate for one example that Pt capping may further enhance the MAE compared to the uncapped JTand the Mackay-distorted $\mathrm{Fe}_{13}$ cluster.
\end{abstract}

DOI: 10.1103/PhysRevB.82.054418 PACS number(s): 75.75. - c, 61.46.Bc, 61.46.Df, 75.30.Gw

\section{INTRODUCTION}

Small transition-metal clusters may be functionalized and used in magnetic nanometer devices. This requires knowledge of the expected spin and orbital magnetic moments as well as of magnetic anisotropy energies. Density functional theory (DFT) is an adequate tool to obtain useful information on the physical properties of small clusters. However, with respect to magnetic properties, the variations in magnetic moments with cluster size and morphology do frequently not allow to establish a clear trend. Also, the exchange coupling in free Fe clusters was found to depend on the cluster size and on the position of the cluster atoms involved in a complex way with no obvious systematics. ${ }^{1,2}$ Still, certain general statements can be made. It may be safely concluded that the local magnetic moments in the outer shells of clusters are enhanced, compared to the interior or to the corresponding bulk crystal. ${ }^{3-6}$ This effect is due to the reduced atomic coordination at the surface and is well confirmed by experiment. ${ }^{7}$ Regarding the influence of structure, an analysis of cluster morphologies, by both experiment and theory, ${ }^{8-14}$ reveals the importance of geometries with icosahedral symmetry, prohibited in periodic structures. The diversity of cluster structures in combination with the surface enhancement of magnetic moments make clusters interesting model objects for tuning magnetic properties at the nanometer scale. ${ }^{15}$
When addressing magnetic properties of clusters from the computational point of view at a realistic level, it is important to take into account two specific issues: (i) a possible noncollinear (NC) setting of magnetic moments (i.e., a smooth variation in the magnetization density vector from point to point in space) and (ii) spin-orbit interaction (SOI), along with the existence of orbital moments. Both issues have a long record of incorporation into first-principles DFT calculations and are internally related: they mix the spin-up and spin-down states and must be, in principle, treated alongside on equal footing (see, e.g., Refs. 16 and 17 for a review). Discussing specifically DFT calculations for clusters, one notes certain technical difficulties in combining NC spin density with the SOI, which acted so far as a limiting factor on the number of calculations performed, and the size of clusters treated: lack of symmetry, big effect of structure relaxation, slow convergence of the calculations, and large size of simulation cell around a free cluster if the method works with periodic boundary conditions. Moreover there is a conceptual problem of choosing a "correct" noncollinear solution among many apparently close metastable configurations. Different groups report very different results for the same systems (e.g., Refs. 18-20) so that a preference of one or another result is not obvious. This means that a clear definition of structural and magnetic models and a careful analysis of their consequences are crucial.

A better understanding of the origin of large orbital magnetic moments and large magnetic anisotropy energies 
(MAE) in clusters ${ }^{21,22}$ is required in order to manipulate material parameters of potential interest, e.g., for magnetic data storage devices. Binary $3 d-5 d$ clusters can be a challenging material in this respect since non magnetic bulk $4 d$ and $5 d$ elements such as Rh, Pt, and Au attain significant moments when alloyed with magnetic $3 d$ transition metals. ${ }^{23-26}$

In the present work, we analyze icosahedral $M_{13}(M$ $=\mathrm{Fe}, \mathrm{Co}, \mathrm{Ni}$ ) clusters and the effect of capping them with $\mathrm{Pt}$ atoms. Several theoretical studies on the magnetic anisotropy of supported clusters ${ }^{15,27-32}$ as well as related studies on small free clusters ${ }^{33-38}$ have recently been published. Recent work on $M_{13}$ clusters $^{39,40}$ focused on the stability and on spin magnetic properties but did not consider spin-orbit effects.

The motivation for the present study is that $M_{13}$ clusters with a structure close to an icosahedral one are known to be very stable $4,6,12$ and that alloying of $3 d$ transition metals with Pt results in large magnetic anisotropy as well as in large orbital moment. ${ }^{41}$ Hence, it is expected that the orbital moments and the MAE of $M_{13}$ clusters will be strongly affected by capping with Pt.

Most of our DFT calculations have been done with the plane-wave code Vienna $a b$ initio simulation package (VASP). ${ }^{42-44}$ For test purposes and for validating the results of calculations on noncapped icosahedral clusters, we performed calculations also with a local-orbitals code, Spanish initiative for electronic simulations with thousands of atoms (SIESTA). ${ }^{45}$ As the two methods are very different in what regards the technical implementation of the DFT calculation scheme, and both have been earlier used in calculations of magnetic clusters, their direct comparison might be of its own interest. In addition, further test calculations for the binary clusters were done using the all-electron local-orbital code full-potential local-orbital scheme (FPLO). ${ }^{46}$ In this respect, it is important to note that the all-electron calculations confirm the results obtained with VASP and SIESTA.

The paper is organized as follows. Section II outlines the computational methods and setup. Section III deals with the results for monometallic icosahedral clusters, notably a comparison between VASP and SIESTA results. Section IV discusses the results for capped clusters, obtained with the VASP code. We have made a comparison between VASP and FPLO for one of the capped clusters. Conclusions are drawn in Sec. V.

\section{COMPUTATIONAL METHODS}

Most of the DFT calculations in the present work were performed with the VASP code, ${ }^{42-44}$ version 4.6 .28 and higher, using the generalized gradient approximation (GGA). The parameterization by Perdew and Wang (PW91) was used for the exchange and correlation functional. ${ }^{47}$ VASP uses the projector augmented wave method ${ }^{44,48}$ and a plane-wavebasis set. Periodic boundary conditions were imposed onto large enough cubic cells with an edge length of $15 \AA$ for $M_{13}$ clusters and $20 \AA$ for $M_{13} \mathrm{Pt}_{n}$ clusters which sufficiently reduces the interaction between replicated cluster images. Since the system of interest is nonperiodic, only the $\Gamma$ point was used for the Brillouin-zone sampling for the cluster calculations. Gaussian broadening for the discrete energy levels

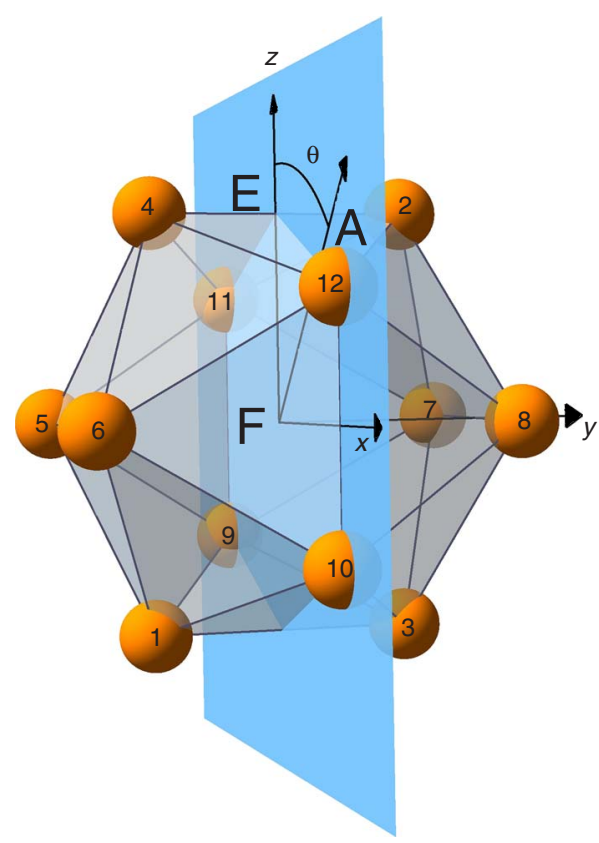

FIG. 1. (Color online) The ideal icosahedral cluster showing the $x-z$ plane in which the angle $\theta$ is varied in the MAE calculations. The same setting was used for partially Mackay-transformed clusters (see below). E, F, and A abbreviate directions from the center to the middle of an edge, the middle of a facet, and an outer atom, respectively. As $\theta$ is varied from 0 to $\pi / 2$, the magnetization direction scans E-F-A-E in succession.

of $0.05 \mathrm{eV}$, a plane-wave cutoff of $270 \mathrm{eV}$, and a Fourier grid spacing of $0.05 \AA^{-1}$ were applied for all calculations except for the MAE calculations, see below. The energy convergence criterion for the self-consistency was set to $10^{-10} \mathrm{eV}$. A $k$ mesh of $(11 \times 11 \times 11)$ divisions and Gaussian broadening as in the cluster calculations was used for the bulk calculations to compute the equilibrium lattice constants of bcc $\mathrm{Fe}$, fcc Ni, and fcc Co. The values for local magnetic moments were obtained by integration of the magnetization density over atom-centered spheres with radii of $1.302 \AA$ (Fe, Co), $1.286 \AA$ (Ni), and $1.455 \AA(\mathrm{Pt})$.

The MAE is defined as the variation in the total energy $E^{\text {tot }}\left(\mathbf{e}_{m}\right)$, as the magnetization takes different orientations $\mathbf{e}_{m}$ $\left(\left|\mathbf{e}_{m}\right|=1\right)$ with respect to the crystalline or molecular frame. Extrema of the MAE are usually but not exclusively found if the magnetization points along a symmetry axis. Thus, it is convenient to orient such an axis parallel to the $z$ axis and to define the reference energy as $E^{\text {tot }}(0,0,1)$. Consequently, the MAE is $\Delta E\left(\mathbf{e}_{m}\right)=E^{\text {tot }}\left(\mathbf{e}_{m}\right)-E^{\text {tot }}(0,0,1)$. In the calculations reported below we made use of the so-called "magnetic force theorem." 49 This means that we approximated the total energy difference by the related difference of single-electron eigenvalue sums, $\Delta E\left(\mathbf{e}_{m}\right) \approx E^{\mathrm{se}}\left(\mathbf{e}_{m}\right)-E^{\mathrm{se}}(0,0,1)$.

In an ideal icosahedron, $\Delta E\left(\mathbf{e}_{m}\right)$ is a highly symmetric function with an irreducible area for the choice of $\mathbf{e}_{m}$ filling only $1 / 120$ of the unit sphere. For this reason we restricted our calculations to a path with a single varying parameter, the polar angle $\theta$, going in such way as to scan through the symmetry axes. Figure 1 depicts our choice for the ideal icosahedron: for $\theta=0$, the magnetization is directed parallel 
to an axis passing through the central atom and the center of a bond connecting two outer atoms; if $\theta$ is gradually increased, the magnetization direction passes through the center of a triangular facet, through an outer atom and finally arrives again at a bond center for $\theta=\pi / 2$. Thus, the path scans all edges of an irreducible segment of the icosahedral surface.

As is well known, the MAE for cubic bulk transition metals is in the order of $10^{-6} \mathrm{eV}$ per atom. Thus, special care is demanded for the study of the MAE both in bulk systems and in clusters because any tiny error can accumulate and produce misleading results while dealing with energy differences of such a small scale. To obtain meaningful results presupposes that the charge density must be extremely well converged and consistent with the symmetry of the system. A distortion of the symmetry might occur due to both numerical fluctuations (which can to a limited extent be controlled by, e.g., the choice of energy cutoff and Fourier grid) and perturbations by the setup of the model system itself, e.g., due to the electrostatic interaction between periodic images of the supercell. If MAE has to be calculated for a relaxed structure (see below), the structural relaxation has to be pursued to sufficiently small values of residual forces on atoms, not exceeding $10^{-6} \mathrm{eV} / \AA$ in our case. In order to calculate the magnetic anisotropy, we used a Gaussian half-width parameter of $0.01 \mathrm{eV}$ for the discrete energy levels. A very high plane-wave cut-off value of $1000 \mathrm{eV}$ as well as a dense Fourier grid spacing of $0.046 \AA^{-1}$ in each direction was taken.

Another difficulty about MAE concerns the treatment of a NC magnetic density. If imposed without constraints and implemented to the point of finding the global minimum of the magnetic state, it would contradict the aim of tracing the magnetization direction to extract the MAE. Therefore, our MAE calculations were done perturbatively, using the collinear spin, varying the global spin axis. A more correct approach would have been to enforce the convergence of $\mathrm{NC}$ magnetic structures in a series of relativistic calculations, repeated for a sequence of external magnetic fields along $\mathbf{e}_{m}$. The possible effect of a truly NC spin density was tested for two systems, $\mathrm{Fe}_{13}$ and $\mathrm{Co}_{13}$, in calculations starting from a collinear (parallel to [001]) spin density. The NC spin and orbital moments were compared to those obtained, for the same cluster geometry and the same initial magnetization, by the SIESTA method, ${ }^{45}$ see Sec. III B.

Differently from VASP, SIESTA uses localized atomcentered basis functions. These functions were generated to the "double- $\zeta$ with polarization orbitals" quality (and triple- $\zeta$ for $3 d$ functions) according to the standard scheme of the SIESTA method, ${ }^{50}$ version 1.4 .13 , with the "energy shift" parameter, which controls the localization of basis functions taken 0.01 Ry. Norm-conserving pseudopotentials of Troullier and Martins ${ }^{51}$ were used; the calculations were done using the GGA, with the parameterization of the exchangecorrelation functional after Perdew, Burke, and Ernzerhof. ${ }^{52}$ The treatment of the SOI was included as described by Fernandez-Seivane et al. $^{53}$

The starting point of all calculations, for the MAE in collinear spin setting as well as for the NC magnetic treatment by VASP and by SIESTA, was a structural relaxation of the clusters, done by VASP in the scalar relativistic mode and using a conjugate-gradient algorithm. From there on, spinorbit coupling was included everywhere, but without any further geometry relaxation.

Finally, for the sake of additional verification, some extra calculations have been done with yet another first-principles calculation method, namely, the all-electron local-orbital FPLO 6.00-24 code $^{46}$ in its cluster mode ${ }^{54}$ using, differently from VASP and SIESTA, the local spin density approximation (LSDA) (Ref. 55) after Perdew and Wang. These calculations are documented later in Sec. IV. The valence basis comprised $3 s 3 p 3 d 4 s 4 p 4 d 5 s$ states for Ni while $5 s 5 p 5 d 6 s 6 p 6 d 7 s$ states were used for Pt. The fully relativistic mode was employed here, where FPLO solves the four-component Dirac-KohnSham equations including spin-orbit coupling in all orders. ${ }^{56}$ Default settings were used for the numerical parameters.

\section{RESULTS FOR MONOMETALLIC ICOSAHEDRAL CLUSTERS $M_{13}$}

\section{A. Structural distortions}

The perfect icosahedral (ICO) $M_{13}$ clusters develop large spin moments and a tendency for a symmetry-lowering distortion, apparently due to a high degeneracy of $d$ levels. We first consider the spin magnetism before structural distortions are addressed. Fixed spin moment calculations show that the $\mathrm{Fe}_{13}$ ICO has two almost degenerate energy minima corresponding to a low-spin state with $34 \mu_{B}$ / cluster (local minimum, the central spin is reversed with respect to the spins of the surface atoms), and a high-spin state with $44 \mu_{B}$ /cluster (global minimum, parallel alignment of all atomic spins). ${ }^{11,35}$ For $\mathrm{Co}_{13}$ and $\mathrm{Ni}_{13}$, we find stable spin magnetic states with $31 \mu_{B}$ /cluster and $8 \mu_{B}$ /cluster, respectively. This result for $\mathrm{Co}_{13}$ differs from the findings by Ma et al.,${ }^{57}$ who find a spin state with $25 \mu_{B}$ /cluster as global minimum for the $\mathrm{Co}_{13}$ icosahedron. Their calculations however employed somewhat a different functional (B3LYP). We performed a related check and found the state with $25 \mu_{B}$ per $\mathrm{Co}_{13}$ cluster at $0.24 \mathrm{eV}$ higher energy than the ground state.

Structural relaxation for the $\mathrm{Fe}_{13}$ cluster is carried out in the high spin state of $44 \mu_{B}$ /cluster. In order to interpret the behavior of a perfect $\mathrm{Fe}_{13}$ ICO we also considered the case of $46 \mu_{B}$ /cluster, which does not show the mentioned high degeneracy of the $d$ levels in the minority spin channel at the Fermi level. This degeneracy, which may also be responsible for the structural distortion, is hindering the accurate evaluation of the MAE, as it is strongly slowing down the numerical convergence.

In order to systematically pursue a search toward probable relaxation patterns from the ideal ICO structure, we "drove" the structure along two different paths, which are known to be relevant from previous studies ${ }^{12,58-60}$ and are likely to lead to different stable or metastable arrangements. These paths are, on the one hand, a Jahn-Teller-type distortion (JT), and, on the other hand, a distortion along the Mackay transformation (MT) path. The relaxation pattern for both transformations is shown in Fig. 2; note that the spatial orientation of clusters is different, in order to underline the remaining symmetry in each case. According to these different orientations, the $\theta$ path in the subsequent discussion of 

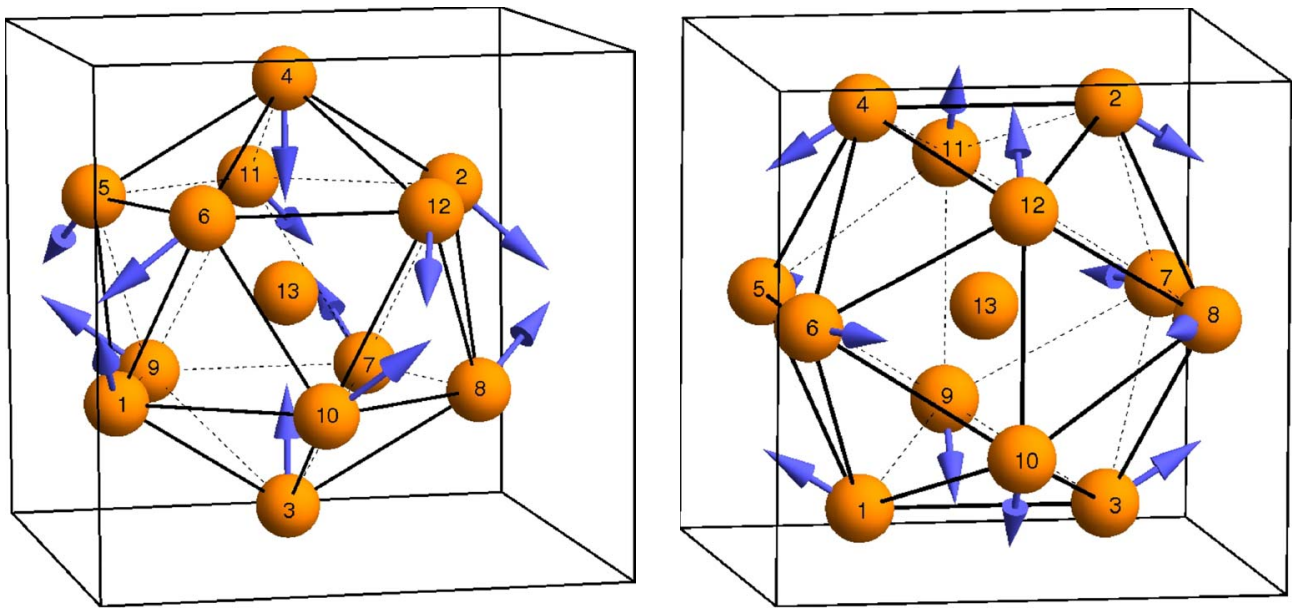

FIG. 2. (Color online) The JT-distorted (left) and the MT (right) $\mathrm{Fe}_{13}$ cluster. Arrows indicate the direction of relative shift of atoms with respect to the ideal positions. For the JT-distorted and $\mathrm{MT} \mathrm{Fe}_{13}$ cluster, the displacements marked by the arrows have been scaled up by factors of 20 and 30, respectively. The box is only guide to the eyes. (The actual simulation box size is $15^{3} \AA^{3}$.)

the MAE is not identical for the two distortions. For the MT one, it follows the previously discussed case of a perfect icosahedron, E-F-A-E for $\theta=[0, \pi / 2]$. For the JT distortion, the $\theta=[0, \pi]$ path is roughly A-E-A-F-E-F-A (see Fig. 2, left panel), slightly deviating from the intermediate symmetric points due to relaxation. As an example for such distortions, we have listed the relaxed coordinates for JT- and MTdistorted $\mathrm{Fe}_{13}$ cluster in Table I.

The JT and the Mackay distortions reduce the $I_{h}$ symmetry of a perfect icosahedron in two different ways, lifting the (quasi-) degeneracy of the highest occupied molecular orbitals and gaining in energy from their splitting. The JT distortion ${ }^{61}$ maintains the fivefold rotational symmetry, permitting a compression or tension of the cluster along the corresponding axis; it may as well involve a mutual opposite rotation of the two pentagonal rings pierced by the axis in question.
The Mackay distortion reduces the $I_{h}$ symmetry to the cubic $T_{h}$ one so that the icosahedral-shell sets on the way of transformation into the fcc cuboctahedron (CUBO), which can be further modified (e.g., along the Bain path) into a bcc-type structure. On the transformation into CUBO, the adjacent triangular facets become pairwise coplanar and join to form squares. This can be numerically characterized by a parameter $s$, defined as the square of the ratio of stretched to unstretched edges, which varies from 1 for ICO to 2 for CUBO (see Ref. 62 for the original explanation). In our calculations, the Mackay distortion parameter is defined as $s=\left|\underline{r}_{4}-\underline{r}_{2}\right|^{2} /\left|\underline{r}_{4}-\underline{r}_{12}\right|^{2}$, where the labeling of atoms is shown in the right panel of Fig. 2.

In Fig. 3, the variation in energy as a function of $s$ (varying from 1 to 2 ) is shown for $\mathrm{Fe}_{13}$ with different total spin magnetic moments. The energy difference is taken with respect to the JT-distorted cluster, which is energetically more

TABLE I. Relaxed coordinates $(\AA)$ for JT-distorted and MT Fe $\mathrm{H}_{13}$ clusters. See Fig. 2 for an illustration and labeling of atoms. Note, that the coordinate axes are not parallel to the cube in the JT case (left panel of Fig. 2).

\begin{tabular}{|c|c|c|c|c|c|c|}
\hline \multirow[b]{2}{*}{ Atom } & \multicolumn{3}{|c|}{$\mathrm{Fe}_{13}(\mathrm{JT})$} & \multicolumn{3}{|c|}{$\mathrm{Fe}_{13}(\mathrm{MT})$} \\
\hline & $x$ & $y$ & $z$ & $x$ & $y$ & $z$ \\
\hline 1 & -0.6304 & -2.0962 & -1.0306 & 0.0000 & -1.2917 & -2.0157 \\
\hline 2 & 0.7221 & 2.0664 & 1.0306 & 0.0000 & 1.2917 & 2.0157 \\
\hline 3 & 0.0000 & 0.0000 & -2.3361 & 0.0000 & 1.2916 & -2.0157 \\
\hline 4 & 0.0000 & 0.0000 & 2.3361 & 0.0000 & -1.2916 & 2.0157 \\
\hline 5 & -1.7988 & -1.2473 & 1.0306 & -1.2917 & -2.0157 & 0.0000 \\
\hline 6 & 0.6304 & -2.0962 & 1.0306 & 1.2916 & -2.0157 & 0.0000 \\
\hline 7 & -0.7221 & 2.0664 & -1.0306 & -1.2916 & 2.0157 & 0.0000 \\
\hline 8 & 1.7421 & 1.3253 & -1.0306 & 1.2917 & 2.0157 & 0.0000 \\
\hline 9 & -2.1884 & -0.0482 & -1.0306 & -2.0157 & 0.0000 & -1.2917 \\
\hline 10 & 1.7988 & -1.2473 & -1.0306 & 2.0157 & 0.0000 & -1.2916 \\
\hline 11 & -1.7421 & 1.3253 & 1.0306 & -2.0157 & 0.0000 & 1.2916 \\
\hline 12 & 2.1884 & -0.0482 & 1.0306 & 2.0157 & 0.0000 & 1.2917 \\
\hline 13 & 0.0000 & 0.0000 & 0.0000 & 0.0000 & 0.0000 & 0.0000 \\
\hline
\end{tabular}




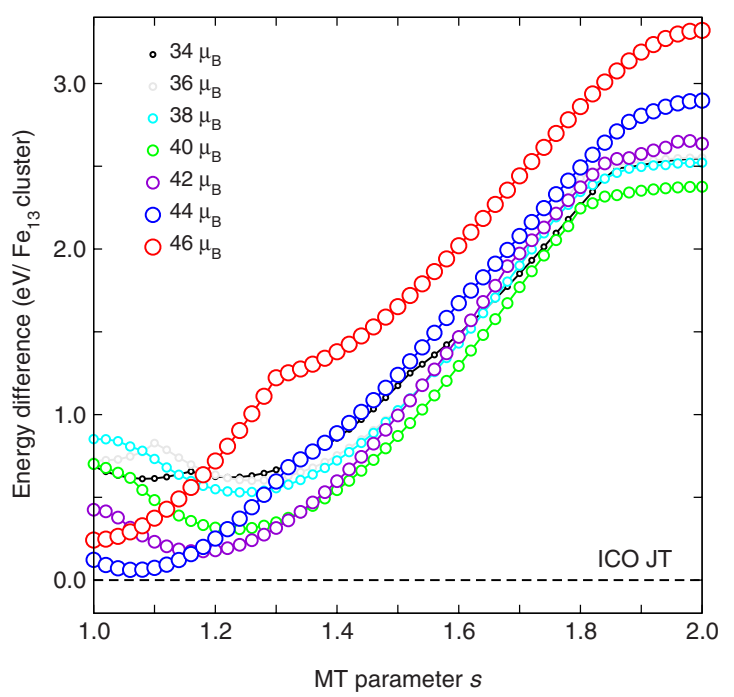

FIG. 3. (Color online) Fixed spin moment results for $\mathrm{Fe}_{13}$ showing the variation in energy as a function of $s$ from ICO $(s=1)$ to CUBO $(s=2)$. The energy difference is given with respect to the lowest energy JT-distorted $\mathrm{Fe}_{13}$ cluster.

favorable compared to all MT-distorted clusters. During these calculations, the distance between center and shell atoms has been kept fixed to $2.39 \AA$, which corresponds to the optimum distance of the ground spin state $\left(44 \mu_{B}\right)$. For several values of the fixed spin moment, kinks appear in the $\mathrm{E}(\mathrm{s})$ curves. This is related to the fact that the fixed spin moment does not completely determine the magnetic state of a system with several inequivalent atomic positions. At the kink positions, two $\mathrm{E}(\mathrm{s})$ curves belonging to different states with the same spin moment cross each other.

For large Fe clusters (beyond cluster sizes of approximately 150 atoms), the JT mechanism does not produce distortions that lead to a significant decrease in energy while the partially MT clusters are characterized by a well-separated minimum on the potential energy surface. ${ }^{12,60}$ This owes to their bcc-like environment in subsurface shells, which effec-
TABLE II. The bond lengths $(\AA)$ for the minimum energy JTdistorted $\left(r=0.965\right.$ for $\mathrm{Fe}_{13}, 1.01$ for $\mathrm{Co}_{13}, 0.98$ for $\left.\mathrm{Ni}_{13}\right)$ and Mackay-transformed ( $s=1.07$ for $\mathrm{Fe}_{13}, 0.96$ for $\mathrm{Co}_{13}, 1.04$ for $\mathrm{Ni}_{13}$ ) $M_{13}$ clusters.

\begin{tabular}{lccc}
\hline \hline Bonds & $\mathrm{Fe}_{13}(\mathrm{JT})$ & $\mathrm{Co}_{13}(\mathrm{JT})$ & $\mathrm{Ni}_{13}(\mathrm{JT})$ \\
\hline $2 \times$ center shell & 2.34 & 2.35 & 2.28 \\
$10 \times$ center shell & 2.42 & 2.32 & 2.33 \\
Bonds & $\mathrm{Fe}_{13}(\mathrm{MT})$ & $\mathrm{Co}_{13}(\mathrm{MT})$ & $\mathrm{Ni}_{13}(\mathrm{MT})$ \\
\hline Centre shell & 2.39 & 2.33 & 2.32 \\
$24 \times$ bond length (surface) & 2.50 & 2.46 & 2.43 \\
$6 \times$ bond length (surface) & 2.58 & 2.41 & 2.47 \\
\hline \hline
\end{tabular}

tively lowers their energy while the core remains unaffected. Molecular-dynamics simulations using embedded atom potentials show, however, that the partially Mackaytransformed clusters are in a metastable ${ }^{63}$ (local energy minimum) state up to cluster sizes with 15 closed atomic shells $\left(n_{k}\right)$ and magic atom numbers $(N)$ defined by

$$
N=\frac{1}{3}\left(10 n_{k}^{3}+15 n_{k}^{2}+11 n_{k}+3\right) \text {. }
$$

The most stable configuration found for clusters of such a size is a bcc-like structure. ${ }^{12}$

Figure 4 shows the variation in energy as a function of the JT parameter $r$ (left panel) defined as $r=\left|\underline{r}_{3}-\underline{r}_{4}\right| /\left|\underline{r}_{1}-\underline{r}_{2}\right|$ with the labeling of atoms shown in the left panel of Fig. 2 and of the Mackay parameter $s$ (right panel) for $M_{13}$ clusters. As the starting structure, we have taken the minimum energy centershell distances for the perfect ICO of $\mathrm{Fe}_{13}, \mathrm{Co}_{13}$, and $\mathrm{Ni}_{13}$, which are $2.39 \AA, 2.33 \AA$, and $2.32 \AA$, respectively. The cluster is now transformed along JT and MT paths and the structural parameters for the local energy minima are compiled in Table II. One must note that in the JT transformation two of the axial atoms have different center-shell distances
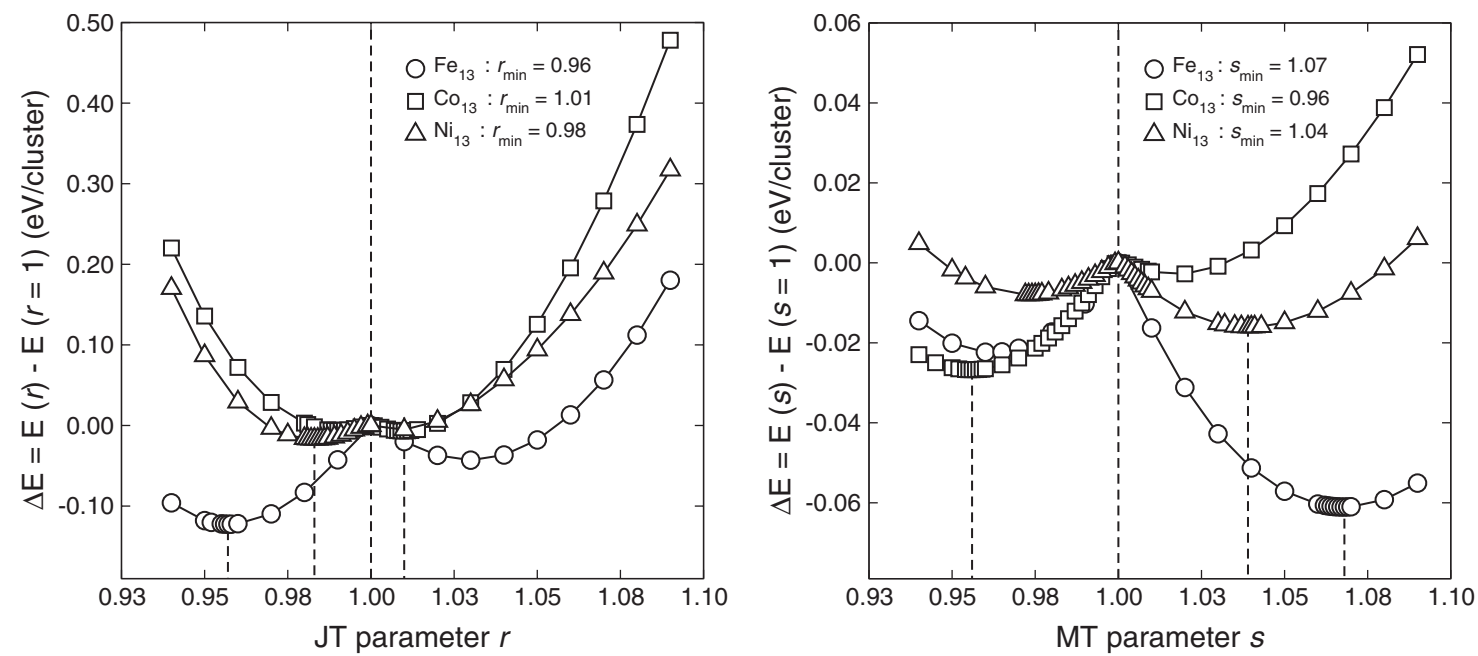

FIG. 4. The minimum energy $r$ and $s$ values obtained for $\mathrm{M}_{13}$ clusters. The dotted lines mark the minimum points for both cases. $s=1$ and $r=1$ represent the perfect ICO for each case. 
TABLE III. Spin and orbital moments of the relaxed $\mathrm{Fe}_{13}(\mathrm{MT})$ cluster as calculated by VASP and SIESTA, for an initial [001] setting of the spin direction. The $x$ and $y$ components of the spin moment are $<0.001 \mu_{B}$, hence not shown here. For the SIESTA calculations, the values of spin moments are given in terms of Mulliken populations and by direct integration of the spin density over atom-centered spheres with a radius of $1.302 \AA$ (latter values are shown in parentheses; the same radius is used in the VASP calculations). The magnetic moments are given in units of $\mu_{B} /$ atom.

\begin{tabular}{|c|c|c|c|c|c|c|c|c|}
\hline \multirow[b]{3}{*}{ Atom No. } & \multicolumn{8}{|c|}{$\mathrm{Fe}_{13}(\mathrm{MT})$} \\
\hline & \multicolumn{4}{|c|}{ VASP } & \multicolumn{4}{|c|}{ SIESTA } \\
\hline & $L_{x}$ & $L_{y}$ & $L_{z}$ & $S_{z}$ & $L_{x}$ & $L_{y}$ & $L_{z}$ & $S_{z}$ \\
\hline 1 & 0.00 & -0.02 & 0.11 & 3.06 & 0.00 & -0.01 & 0.09 & $3.43(3.14)$ \\
\hline 2 & 0.00 & 0.02 & 0.12 & 3.06 & 0.00 & 0.01 & 0.09 & $3.43(3.12)$ \\
\hline 3 & -0.01 & 0.00 & 0.08 & 3.08 & -0.01 & 0.00 & 0.08 & $3.43(3.16)$ \\
\hline 4 & 0.01 & 0.00 & 0.08 & 3.08 & 0.01 & 0.00 & 0.08 & $3.44(3.12)$ \\
\hline 5 & 0.00 & 0.00 & 0.07 & 3.10 & 0.00 & 0.00 & 0.06 & $3.44(3.18)$ \\
\hline 6 & 0.00 & 0.00 & 0.07 & 3.10 & 0.00 & 0.00 & 0.06 & $3.44(3.15)$ \\
\hline 7 & 0.00 & 0.00 & 0.07 & 3.10 & 0.00 & 0.00 & 0.06 & $3.44(3.16)$ \\
\hline 8 & 0.00 & 0.00 & 0.07 & 3.10 & 0.00 & 0.00 & 0.06 & $3.44(3.13)$ \\
\hline 9 & 0.01 & 0.00 & 0.08 & 3.08 & 0.01 & 0.00 & 0.08 & $3.44(3.18)$ \\
\hline 10 & -0.01 & 0.00 & 0.08 & 3.08 & -0.01 & 0.00 & 0.08 & $3.44(3.15)$ \\
\hline 11 & 0.00 & 0.02 & 0.11 & 3.05 & 0.00 & 0.01 & 0.09 & $3.43(3.18)$ \\
\hline 12 & 0.00 & -0.02 & 0.11 & 3.06 & 0.00 & -0.01 & 0.09 & $3.43(3.16)$ \\
\hline 13 & 0.00 & 0.00 & 0.05 & 2.70 & 0.00 & 0.00 & 0.03 & $2.75(2.61)$ \\
\hline
\end{tabular}

compared to the other atoms. However, for MT, the centershell distances are independent of $s$. The MAE of the local energy-minima JT and MT clusters are calculated and are discussed in Sec. III D.

The energy gain due to the relaxation from the perfect ICO structure for $\mathrm{Fe}_{13}$ (center-shell distance $2.39 \AA$ ) is 125 $\mathrm{meV} /$ cluster along the JT distortion and $61 \mathrm{meV} /$ cluster along the MT, with $44 \mu_{B}$ spin magnetic moment for both. The existence of two different metastable relaxed structures for this cluster has been earlier reported in Refs. 12, 58, and 59. For $\mathrm{Co}_{13}$, the JT relaxation is by $7 \mathrm{meV} /$ cluster and the MT relaxation is by $27 \mathrm{meV}$ lower in energy with respect to the perfect ICO, whereas for $\mathrm{Ni}_{13}$ the energy differences are nearly the same ( $16 \mathrm{meV} /$ cluster) for both JT and MT clusters, respectively. We have only considered the icosahedral symmetry of $\mathrm{Co}_{13}$ cluster for our studies but a layered hep structure is found to be the ground state for this cluster. ${ }^{40}$

At this place it seems appropriate to take note of recent results by Ma et al. on small transition-metal clusters, including $\mathrm{Fe}_{13}, \mathrm{Co}_{13}$, and $\mathrm{Ni}_{13}$. The related calculations were carried out with a so-called hybrid functional (B3LYP) different from the GGA approach used in our calculations. Thus, we do not aim at a direct comparison but rather mention the main results on $\mathrm{M}_{13}$ clusters. For $\mathrm{Fe}_{13}$, Ma et al. ${ }^{64}$ find a ground state with $D_{2 h}$ symmetry and $\mu_{s}=44 \mu_{B}$ about 90 meV below the ideal ICO with the same spin moment. For $\mathrm{Co}_{13}$, they find a ground state with $D_{2 h}$ symmetry and $\mu_{s}$ $=23 \mu_{B}$ about $820 \mathrm{meV}$ below the ideal ICO with $\mu_{s}$ $=25 \mu_{B} \cdot{ }^{57}$ While for $\mathrm{Ni}_{13}$, the same authors find a ground state with $D_{3 d}$ symmetry and $\mu_{s}=8 \mu_{B}$ about $660 \mathrm{meV}$ below the ideal ICO with the same spin moment. ${ }^{65}$
We now turn back to Table I which lists the relaxed coordinates for the JT- and MT-distorted $\mathrm{Fe}_{13}$ clusters. For the JT-distortion, ten of the peripheral atoms lie at a distance of $2.42 \AA$ from the center, the other two atoms show an inward relaxation toward the center with a final distance of $2.34 \AA$ from the center. The peripheral bond lengths for the MT clusters (see Table II) can be arranged in two groups: six of the 30 bonds parallel to the cartesian axes $(x: 5-6,7-8 ; y$ : $1-3,4-2 ; z: 9-11,10-12$; see Fig. 2 for the labeling of the atoms), and the remaining 24 bonds. By symmetry, the distances of the 12 peripheral atoms to the central one are all identical. The structural relaxation of the $\mathrm{Fe}_{13}$ cluster shown in the right panel in Fig. 2 corresponding to the partial Mackay transformation yields $s=1.07 ; \mathrm{Co}_{13}$ and $\mathrm{Ni}_{13}$ have $s=0.96$ and 1.04 , respectively.

\section{B. Spin and orbital magnetic moments}

The relaxed MT coordinates obtained with VASP have been used, without further relaxation, in the calculation by SIESTA, in order to compare the resulting values of spin and orbital moments, and the noncollinearity. Table III compares the calculated results by the two methods for the $\mathrm{Fe}_{13}$ (MT) cluster.

For both methods, the spin moments remain parallel (the $x$ and the $y$ components of the spin vectors amount to at most $0.001 \mu_{B}$ and are not shown in Table III). The orbital moments partly seem to deviate from the $z$ axis but we note that these deviations are not significantly above the numerical noise level. This becomes evident if one compares data for quadruples of atoms that are mutually equivalent (apart from 
TABLE IV. Cluster averaged values for $\langle L\rangle=\frac{1}{13} \sum_{i=1}^{13}\left|\mathbf{L}_{i}\right|$ and $\langle S\rangle=\frac{1}{13} \sum_{i=1}^{13}\left|\mathbf{S}_{i}\right|$ of MT $M_{13}$ clusters are shown in $\mu_{B} /$ atom, compared to bulk values for bcc Fe, fcc $\mathrm{Co}$ and $\mathrm{Ni}$ as obtained from our calculations.

\begin{tabular}{lcccc}
\hline \hline Cluster & $\langle L\rangle$ & $\langle S\rangle$ & $\langle|\mathbf{L}|\rangle_{\text {bulk }}$ & $\langle|\mathbf{S}|\rangle_{\text {bulk }}$ \\
\hline $\mathrm{Fe}_{13}$ (MT) & 0.08 & 3.05 & 0.06 & 2.25 \\
$\mathrm{Co}_{13}$ (MT) & 0.12 & 2.05 & 0.08 & 1.67 \\
$\mathrm{Ni}_{13}$ (MT) & 0.06 & 0.66 & 0.05 & 0.65 \\
\hline \hline
\end{tabular}

rotations around the $z$ axis) by symmetry: $1-4,5-8$, and 9-12. While the symmetry requirement is (almost) obeyed by the atoms 5-8, deviations of a few hundredth $\mu_{B}$ are found between the atoms $1-4$ and also between the atoms 9-12.

When comparing the numerical results for spin and orbital magnetic moments from these two different calculation methods, one must take into account the difference in their definitions. In VASP, the properties (spin and orbital moments) are extracted as projection onto an atomic sphere (see Sec. II). The "standard" VASP value of the atomic sphere radius for both considered systems does in fact correspond to slightly overlapping spheres. The SIESTA output results are reported in terms of decomposition by projection onto localized, but overlapping, numerical orbitals, known as Mulliken population analysis. It is known that the local magnetic moments as well as the atomic charges in heterogeneous systems do often come out very different, when estimated according to these two different schemes. In order to illustrate this effect, we give in the last column of Table III (in parentheses), the values of spin moment, extracted from the SIESTA results by summing up the spatial spin density over atomcentered spheres with a radius of $1.302 \AA$. The fluctuations of these integrated values over apparently equivalent atoms are caused by the sparseness of the spatial grid with steps of $0.078 \AA$, on which the spin density summation has been done. While the results of the Mulliken population analysis by definition add up to the total moments, the added values of the spatial summations are smaller than the total moments by about $10 \%$.

We note, as expected, a significant difference between the spin moments of central and peripheral atoms. This difference can be used to check the influence of different projection schemes. Taking a spatial summation of the spin density, the difference between the two kinds of atoms amounts to about $0.4 \mu_{B}$ (VASP) and $0.5 \mu_{B}$ (SIESTA) for $\mathrm{Fe}_{13}$. If the Mulliken analysis is taken (SIESTA), different values of about $0.7 \mu_{B}$ for $\mathrm{Fe}_{13}$ are found. It is not possible to assign a general preference to any of the schemes but we would like to stress that such different evaluation schemes may give rise to different interpretations. In the Appendix, we show a comparison of orbital and spin moments for $\mathrm{Co}_{13}$ and $\mathrm{Ni}_{13}$ clusters calculated with the two methods.

The cluster averaged orbital moments $\langle L\rangle$ and the cluster averaged spin moments $\langle S\rangle$ of $\mathrm{Fe}_{13}, \mathrm{Co}_{13}$, and $\mathrm{Ni}_{13}$ clusters are compared with related bulk values in Table IV. The orbital and spin moments for the bulk systems are calculated at the equilibrium lattice constants of $2.83 \AA$ (bcc Fe), $3.52 \AA$ (fcc Co), and $3.523 \AA$ (fcc Ni). The spin magnetic moments of the elemental $\mathrm{Fe}$ and Co clusters are larger than the related bulk values. As discussed in the Introduction, such an enhancement is expected for small clusters. The $\mathrm{Ni}_{13}$ spin moment is approximately the same as the related bulk value.

The orbital moments of all three elements are found to be larger by factors of $\sim 1.2-1.5$ in the cluster geometry than in the bulk. For $\mathrm{Co}$ and $\mathrm{Ni}$, the orbital magnetic moments are yet more sensitive to the geometry than spin magnetic moments. A related tight-binding model calculation ${ }^{66}$ found that in Ni clusters of up to 13 atoms, the average orbital moment $\langle L\rangle$ per atom is four to eight times larger than the bulk value; for larger cluster sizes, $\langle L\rangle$ was shown to approach the bulk value. The enhancement reported for small clusters is much larger than our related GGA value, since an LSDA $+U$-like approach was used in Ref. 66, considering the effect of local correlations.

\section{MAE of perfect icosahedral clusters}

Cubic bulk $3 d$ metals show a tiny MAE in the order of $\sim 1 \mu \mathrm{eV}$. The tiny value in cubic systems is due to the high symmetry and it is expected that an ideal ICO exhibits a similarly small MAE. If the symmetry is broken, e.g., by tetragonal distortion of the cubic system, the MAE increases considerably (see, for instance, Ref. 67). Such an enhancement of the MAE is also expected for distorted ICO structures.

Figure 5 (left panel) shows the computed MAE of a perfect ICO $\mathrm{Fe}_{13}$ cluster in comparison with a simple classical expansion of $\Delta E(\theta)$ following the Néel model. Convergence problems due to the dense level sequence close to the Fermi level hindered a calculation for the ground spin state (44 $\mu_{B}$ ). Thus, the data presented were obtained for a state with a spin moment of $46 \mu_{B}$ and a related center-shell distance of $2.57 \AA$. As discussed in Sec. II, extrema of the $\Delta E(\theta)$ dependence are found for $\theta$ values related to symmetry axes, see Fig. 1. In the present case, global maxima (hard axes) are obtained if the magnetization is directed parallel to a line from the central atom to one of the twelve atoms of the outer shell. Global minima (easy axes) are obtained if the magnetization is directed parallel to a line from the central atom to the middle point of one of the twenty facets. The 30 symmetric mid-bonds are, consequently, saddle points of the anisotropy energy. The magnitude of the energy variation per atom amounts to $1.7 \mu \mathrm{eV}$, comparable to that in bulk bcc Fe. In the right panel of Fig. $5, \Delta E$ is shown in the whole $(\theta, \phi)$ plane.

In addition, the MAE for perfect ICO $\mathrm{Co}_{13}$ and $\mathrm{Ni}_{13}$ clusters are calculated to be $0.31 \mu \mathrm{eV} /$ atom and $0.77 \mu \mathrm{eV} /$ atom, respectively, for a center-shell distance of $2.33 \AA\left(\mathrm{Co}_{13}\right)$ and $2.32 \AA\left(\mathrm{Ni}_{13}\right)$. These calculations start from states with spin moments of $31 \mu_{B}$ /cluster for $\mathrm{Co}_{13}$ and $8 \mu_{B}$ /cluster for $\mathrm{Ni}_{13}$. The qualitative $\theta$ dependence of $\Delta E$ agrees with that of the perfect ICO $\mathrm{Fe}_{13}$.

The GGA results were fitted to the anisotropy expansion of a classical spin model, following the Néel surface anisotropy model ${ }^{68,69}$ for a nearly spherical cluster: 

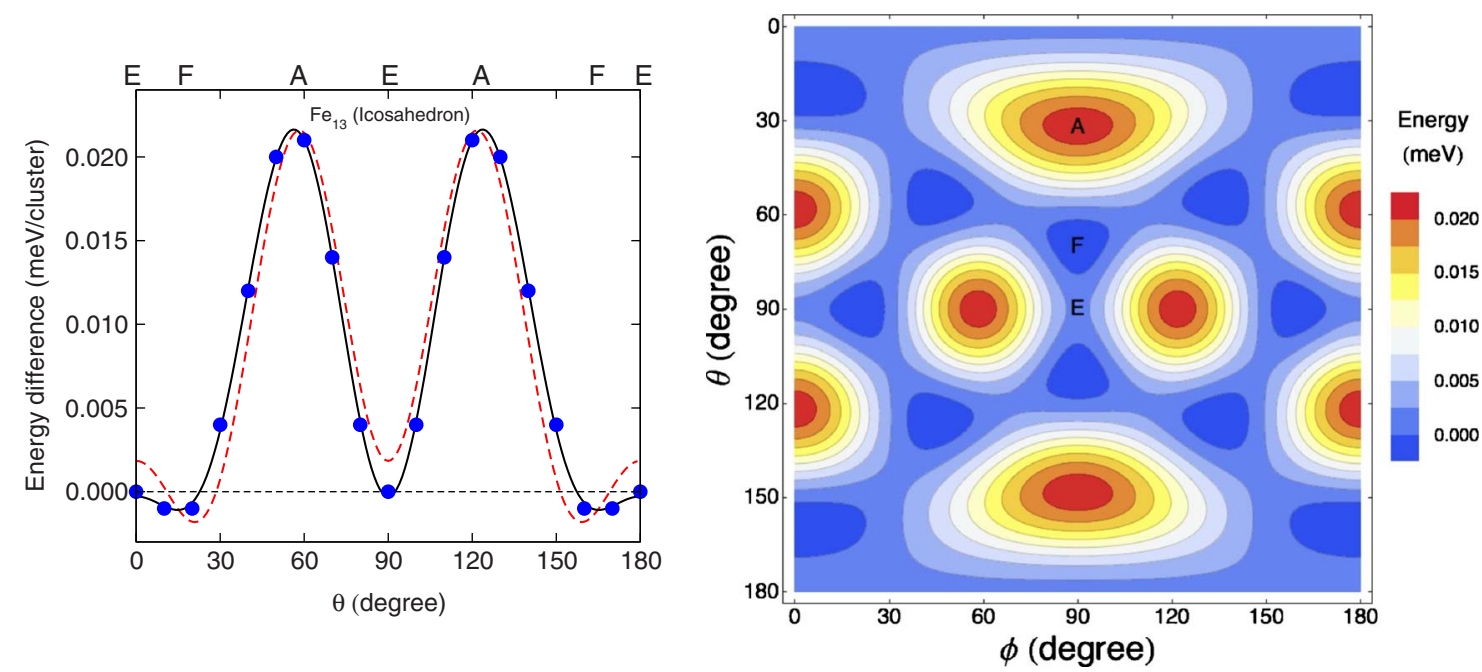

FIG. 5. (Color online) Left: the $\theta$-dependent energy (in meV per cluster) for an ideal $\mathrm{Fe}_{13}$ icosahedral cluster (state with a spin moment of $46 \mu_{B}$ and a center-shell distance of $2.57 \AA$ ). Filled circles: GGA results (VASP calculations), connected by a solid line as guide to the eye. Dashed line: fit to the anisotropy term according to Eq. (1), for $n=6$. For the vasP calculations, the energy difference is defined as $\Delta E$ $=E(\theta)-E(0)$. The letters $\mathrm{A}, \mathrm{E}$, and $\mathrm{F}$ in the plot refer to the positions defined in Fig. 1. Right: the $(\theta, \phi)$ scan for the MAE of an ideal Fe 13 cluster: The energies plotted are the sixth-order contributions to the total energy using the value of $D_{6}$ from Table V.

$$
\Delta E_{\text {Néel }}=\sum_{n} E_{n}=-\sum_{n} \sum_{i=1}^{N} D_{n}\left(\mathbf{e}_{i} \cdot \mathbf{e}_{m}\right)^{n}
$$

Here, $D_{n}$ is the anisotropy constant of order $n$ ( $n$ being an even integer) and $\mathbf{e}_{i}$ is the normalized position vector of atom $i$, taking the central atom as origin. The $i$ summation runs only over the peripheral atoms, and the $D_{n}$ are $\theta$ independent by definition. The second- and fourth-order contributions are constant from symmetry considerations for an ideal ICO. Figure 5 shows a comparison of GGA results with a leastmean square fit to $\Delta E_{\text {Néel }}$ taking into account only the $n=6$ term, see Table V. While all higher terms $n=8, \ldots$ in principle contribute to the $\theta$ dependence for a perfect ICO, the comparison shows that these terms are negligibly small and a sixth-order fit provides a very good description.

\section{MAE of distorted clusters}

The MAE $\Delta E(\theta)$ for relaxed clusters is shown in Fig. 6. For the JT-distorted $M_{13}$ clusters (left panel of Fig. 6), a large second-order contribution of $D_{2}$ is found, since both the icosahedral and the cubic symmetries are broken by the distortion. All higher order terms are significantly smaller and can safely be neglected. In contrast, there is no second-order contribution present in the MT $M_{13}$ clusters due to their cubic symmetry. Again, only the lowest order (here, $D_{4}$ ) matters, and higher orders are not important. The related values are compiled in Table V. The MAE of the JT-distorted $\mathrm{Fe}_{13}$

TABLE V. Anisotropy constants $D_{n}$ according to Eq. (1), obtained by fitting the GGA results for ideal and relaxed $M_{13}$ clusters. In the icosahedral symmetry, second- and fourth-order contributions do not depend on $\theta$ for any value of $D_{2,4}$. Thus, the related data are omitted. The same holds for the second-order terms in cubic symmetry (MT clusters). In all cases, only the leading order terms are essential and all higher order terms can be neglected. $r$ and $s$ are parameters describing the JT and the Mackay transformation, respectively (see text). The last column of the table compiles the GGA energy differences.

\begin{tabular}{|c|c|c|c|c|c|c|c|}
\hline Cluster & & $\begin{array}{c}\mathrm{D}_{2} \\
(\mathrm{meV})\end{array}$ & $\begin{array}{c}\mathrm{D}_{4} \\
(\mathrm{meV})\end{array}$ & $\begin{array}{c}\mathrm{D}_{6} \\
(\mathrm{meV})\end{array}$ & $r-1$ & $s-1$ & $\begin{array}{c}\Delta \mathrm{E}^{\mathrm{DFT}} \\
(\mathrm{meV})\end{array}$ \\
\hline $\mathrm{Fe}_{13}(\mathrm{ICO})$ & $46 \mu_{B}$ & & & -0.04 & 0 & 0 & 0.02 \\
\hline $\mathrm{Co}_{13}(\mathrm{ICO})$ & $31 \mu_{B}$ & & & -0.01 & 0 & 0 & 0.004 \\
\hline $\mathrm{Ni}_{13}(\mathrm{ICO})$ & $8 \mu_{B}$ & & & -0.02 & 0 & 0 & 0.01 \\
\hline $\mathrm{Fe}_{13}(\mathrm{JT})$ & $44 \mu_{B}$ & 15.0 & & & -0.04 & & 4.20 \\
\hline $\mathrm{Co}_{13}(\mathrm{JT})$ & $31 \mu_{B}$ & -16.0 & & & 0.01 & & 1.90 \\
\hline $\mathrm{Ni}_{13}(\mathrm{JT})$ & $8 \mu_{B}$ & 44.1 & & & -0.02 & & 8.90 \\
\hline $\mathrm{Fe}_{13}(\mathrm{MT})$ & $44 \mu_{B}$ & & -11.5 & & & 0.07 & 0.71 \\
\hline $\mathrm{Co}_{13}(\mathrm{MT})$ & $31 \mu_{B}$ & & -0.4 & & & -0.04 & 0.02 \\
\hline $\mathrm{Ni}_{13}(\mathrm{MT})$ & $8 \mu_{B}$ & & -10.1 & & & 0.04 & 0.32 \\
\hline
\end{tabular}



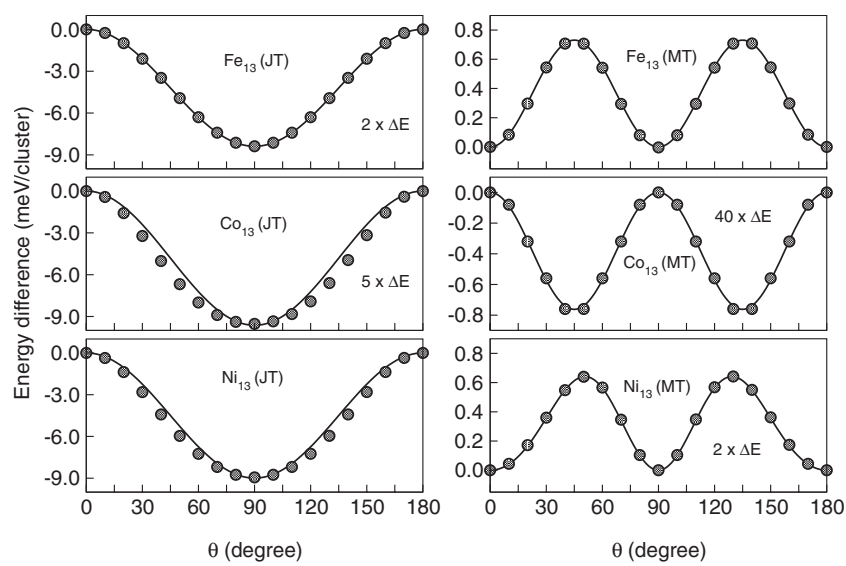

FIG. 6. The plots from top to bottom show the energy differences $\Delta E=E(\theta)-E(0)$ in meV/cluster vs $\theta$ of relaxed $\mathrm{M}_{13}$ (for two types of relaxations-JT and Mackay distortion) clusters. The magnetization direction $(\theta)$ varies in the $x$ - $z$ plane. For the JT case (left panel), this plane passes through atoms 3 and 4 and almost touches atom 12 and the midpoint of the bond 8-10 (see Fig. 2, left panel); for the MT case (right panel), compare Fig. 1. For the JT-distorted $M_{13}$ clusters, the energy difference for the $\mathrm{Co}_{13}$ and $\mathrm{Fe}_{13}$ clusters is multiplied by factors of 5 and 2, respectively, whereas for the MT clusters, the energy difference for $\mathrm{Co}_{13}$ and $\mathrm{Ni}_{13}$ is multiplied by factors of 40 and 2, respectively. The slight deviations between the GGA (circles) and fitted data (full lines) appear for $\mathrm{Co}_{13}(\mathrm{JT})$ and $\mathrm{Ni}_{13}$ (JT) due to the presence of small $D_{4}$ contributions, were neglected.

cluster is calculated to be about $322 \mu \mathrm{eV} / \mathrm{atom}$, which is approximately six times larger in comparison with the MT $\mathrm{Fe}_{13}$ cluster $(55 \mu \mathrm{eV} /$ atom). The reason behind this difference is the lower (higher) symmetry of the JT-distorted (MT) cluster. For $\mathrm{Co}_{13}$ and $\mathrm{Ni}_{13}$, the JT-distorted clusters show larger values of MAE compared to the corresponding Mackay-distorted ones as well. In the present calculation, the MAE value of the JT-distorted $\mathrm{Co}_{13}$ cluster is obtained as $147 \mu \mathrm{eV} /$ atom, which is approximately 100 times larger than the MAE of the MT-distorted cluster (1.42 $\mu \mathrm{eV} /$ atom). Among all clusters (both JT and MT), we obtain the largest value of MAE for the JT-distorted $\mathrm{Ni}_{13}$ cluster (688 $\mu \mathrm{eV} /$ atom). This is approximately 30 times larger than the MAE of the MT-distorted $\mathrm{Ni}_{13}$ cluster.

The data for $\Delta E(\theta)$ for the relaxed $\mathrm{MT} \mathrm{Fe}_{13}$ cluster, see top right panel of Fig. 6 show on a first sight qualitative resemblance with that of the ideal cluster (see Fig. 5). At a glance one notes, however, the additional minimum at the facet centers of the ideal ICO. More important, the MAE of the MT $\mathrm{Fe}_{13}$ cluster is approximately 30 times larger than that of the related ideal cluster. This comparison strikingly underlines the importance of symmetry for the magnetic anisotropy: by a seemingly small JT distortion, the anisotropy of an $\mathrm{Fe}_{13}$ cluster changes by about 2 orders of magnitude. (Note that this comparison is only a qualitative one since we considered the ideal ICO in a different spin state for technical reasons.)

Our calculations yield a larger value of MAE per atom for JT- and Mackay-distorted $\mathrm{Fe}_{13}$ and $\mathrm{Ni}_{13}$ compared to the bulk, where LSDA calculations found $1.4 \mu \mathrm{eV} /$ atom (bcc
Fe), $2.7 \mu \mathrm{eV} /$ atom (fcc Ni). ${ }^{70}$ For $\mathrm{Co}_{13}$, we obtain a different trend: While the JT-distorted $\mathrm{Co}_{13}$ has a MAE value approximately 100 times larger than the related bulk value $(1.3 \mu \mathrm{eV} /$ atom for fcc $\mathrm{Co})$, for Mackay-distorted $\mathrm{Co}_{13}$, it is close to that of the bulk.

The atomic coordinates of the perfect ICO as well as the atomic coordinates along the transformation path from ICO to CUBO can be given in analytical form in terms of the parameter $s$, assuming the conservation of cluster volume with a fixed center to shell distance. Thus, the energy dependence on the structural parameter $s$ and on the anisotropy coefficients can be calculated from a Taylor expansion for a Mackay-transformed cluster around $s=1$ to give

$$
\begin{gathered}
\Delta E_{2}=0, \\
\Delta E_{4}=E_{4}(\mathrm{~A})-E_{4}(\mathrm{E})=D_{4}\left[-\frac{4}{5}(\sqrt{5}-1)(s-1)\right. \\
\left.-\frac{8}{25}(\sqrt{5}-4)(s-1)^{2}+O(s-1)^{3}\right], \\
\Delta E_{6}=E_{6}(A)-E_{6}(\mathrm{~F})=D_{6}\left[-\frac{128}{225}+O(s-1)\right],
\end{gathered}
$$

where $\Delta E_{n}(\mathrm{X})$ is the energy difference between two extrema, labeled $X=\mathrm{A}, \mathrm{E}, \mathrm{F}$ and introduced in Fig. 1.

The leading contribution $\Delta E_{4}$ is linear in $(s-1)$. Thus, the MAE of MT-distorted $\mathrm{Fe}_{13}(s>1)$ and $\mathrm{Co}_{13}(s<1)$ have opposite signs in spite of equal signs of the related coefficients $D_{4}$, see Table V. The large difference of the absolute magnitude of the MAE can only be understood from a detailed analysis of the electronic structure which is beyond the scope of the present work.

Similarly, as mentioned before, a distortion parameter $r$ can be defined for the JT distortion via $r=\left|\underline{r}_{3}-\underline{r}_{4}\right| /\left|\underline{r}_{1}-\underline{r}_{2}\right|$ with $\underline{r}_{n}$ denoting the position of atom $n$ in the left panel of Fig. 2. A Taylor series expansion for a JT distortion under volume conservation (the transformation is volume conserved) around $r=1$ yields

$$
\Delta E_{2}=D_{2}\left[-6(r-1)-\frac{3}{2}(r-1)^{2}+O(r-1)^{3}\right] .
$$

\section{RESULTS FOR CAPPED CLUSTERS $M_{13} \mathrm{Pt}_{n}$}

Obviously, binary clusters show a yet larger diversity compared to the elemental systems. For example, the intermixing of Co with $\mathrm{Rh}$ results in large magnetic moments of the binary systems. ${ }^{26}$ Also, both free FePt clusters and Co clusters supported by a Pt substrate show enhanced spin and orbital moments with respect to corresponding bulk values. ${ }^{71}$ Hence, it is interesting to study how the magnetic properties including the MAE of $M_{13}$ clusters change as they are capped with $\mathrm{Pt}$ atoms. Of particularly great concern is to investigate the evolution of spin and orbital moments as well as the MAE in more "asymmetric" clusters as obtained by adatoms. To this end, we have added a varying number of $\mathrm{Pt}$ atoms on the top of $M_{13}$ clusters and studied the resulting properties. 

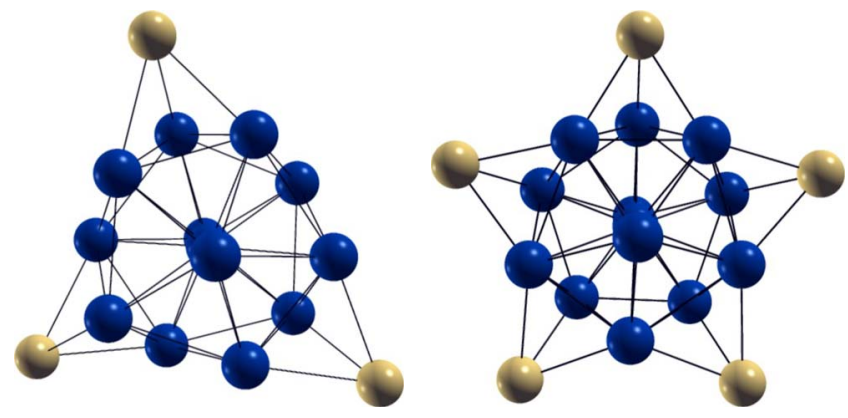

FIG. 7. (Color online) The lowest energy isomers of $\mathrm{Co}_{13} \mathrm{Pt}_{3}$ (left) and of $\mathrm{Co}_{13} \mathrm{Pt}_{5}$ (right), found so far. Dark (blue) spheres: Co atoms; light (yellow) spheres: $\mathrm{Pt}$ atoms. All $\mathrm{Pt}$ atoms of $\mathrm{Co}_{13} \mathrm{Pt}_{5}$ lie in the same plane.

We considered three high-symmetry positions (A, E, and F in Fig. 1) to cap the $M_{13}$ clusters by a single Pt atom and found that a $\mathrm{Pt}$ position above the middle point of a facet is most favorable in all three cases, $M=\mathrm{Fe}, \mathrm{Co}, \mathrm{Ni}$. In the following, we used this finding as a guideline for initial geometries of $M_{13} \mathrm{Pt}_{n}$ clusters $(n=3,5,20)$. In all cases, the $\mathrm{Pt}$ atoms were initially placed above the facet centers at a distance found in the single-Pt capping case. After relaxation, optimized geometries were obtained as exemplarily shown in Fig. 7 for $\mathrm{Co}_{13} \mathrm{Pt}_{3}$ (left) and for $\mathrm{Co}_{13} \mathrm{Pt}_{5}$ (right) and in Fig. 8 (right side) for $\mathrm{Ni}_{13} \mathrm{Pt}_{20}$.

Since atom projected quantities such as spin and orbital moments depend on the specific code, we compared for the particular case of $\mathrm{Ni}_{13} \mathrm{Pt}_{3}$ related data obtained by VASP and FPLO. The structure optimization was carried out by VASP and the same geometry was used to evaluate the magnetic moments by both codes. In FPLO, the magnetic moments are calculated through Mulliken population analysis. Figure 9 shows the absolute value of orbital moment per atom $\left|\mathbf{L}_{i}\right|$ (left) and the absolute value of spin moment per atom $\left|\mathbf{S}_{i}\right|$ (right) on each atomic site. It is obvious that both codes give results which are in good agreement with each other. Therefore, the calculations which are discussed in the following are done with VASP only.

In Figs. 10 and 11, the variation in $\left|\mathbf{L}_{i}\right|$ and the variation in $\left|\mathbf{S}_{i}\right|$ on each atomic site of $M_{13} \mathrm{Pt}_{n}$ clusters are shown, respec-
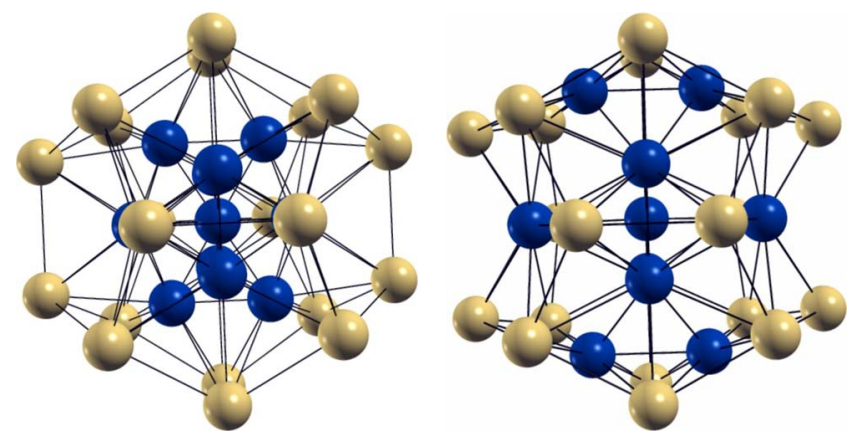

FIG. 8. (Color online) The initial (left) and final (right) structures of the $\mathrm{Ni}_{13} \mathrm{Pt}_{20}$ cluster. $\mathrm{Ni}$ and $\mathrm{Pt}$ atoms are represented by dark (blue) and light (yellow) spheres. The final structure (the lowest energy found so far) demonstrates the importance of atomic relaxations.

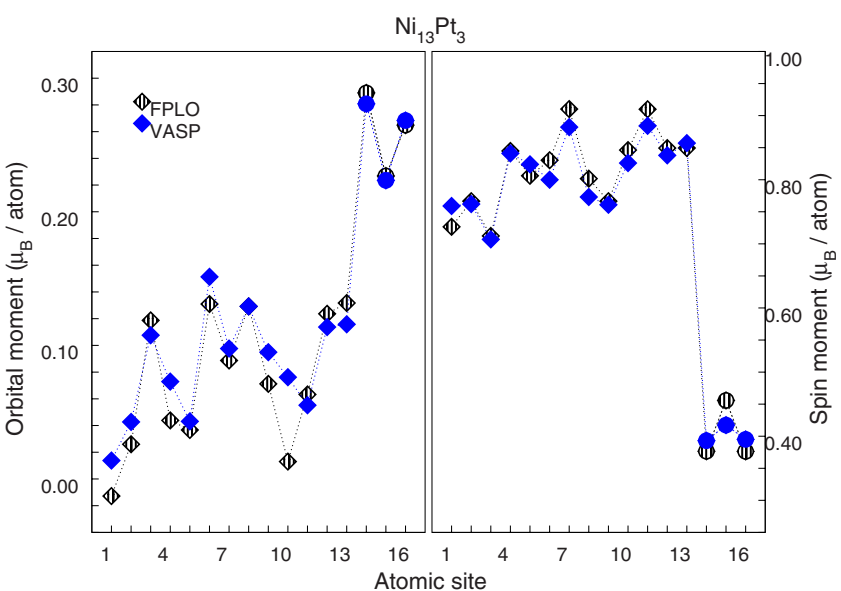

FIG. 9. (Color online) A comparison of onsite orbital moments $\left|\mathbf{L}_{i}\right|$ (left panel) and spin moments $\left|\mathbf{S}_{i}\right|$ (right panel) for the $\mathrm{Ni}_{13} \mathrm{Pt}_{3}$ cluster obtained by VASP and FPLO. In the left panel, the dashed and filled diamonds show the orbital moments of $\mathrm{Ni}_{13}$ cluster obtained with FPLO and VASP while the dashed and filled circles are the orbital moments of three capped Pt atoms, respectively. The right panel shows the corresponding results of the individual spin moments. Atomic site 1 is the center atom.

tively (the symbols are kept consistent for both figures and the center atom is placed at 1). Figure 10 nicely shows that the orbital moments of such few-atom systems in general depend very sensitively on the particular chemical composition and geometry. The resulting electronic structure can be very individual (e.g., the nature of the highest occupied level depends on the electron number and on the spin moment) and is hard or impossible to be predicted without a detailed calculation. It is found that (see Fig. 10) the $\left|\mathbf{L}_{i}\right|$ of $\mathrm{Fe}_{13}$ clusters approach the corresponding bulk value of bcc $\mathrm{Fe}$ reported in Table VI with increasing number of $\mathrm{Pt}$ atoms. This trend is absent in the related $\mathrm{Co}$ and Ni systems (refer to Table VI). The orbital moment on Pt atoms is found to be very sensitive with respect to the core atomic species. For $\mathrm{Fe}$

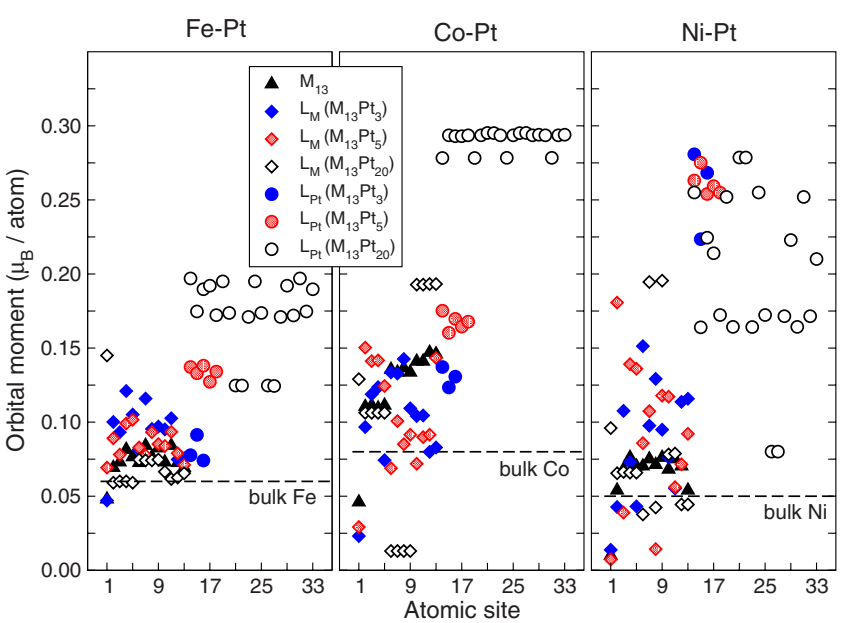

FIG. 10. (Color online) The orbital moment $\left|\mathbf{L}_{i}\right|$ of each atomic site of $\mathrm{Fe}_{13} \mathrm{Pt}_{n}$ (left), $\mathrm{Co}_{13} \mathrm{Pt}_{n}$ (middle), and $\mathrm{Ni}_{13} \mathrm{Pt}_{n}$ (right) clusters. The dashed lines show the calculated bulk values of the orbital moments of bcc $\mathrm{Fe}$, fcc $\mathrm{Co}$, and fcc $\mathrm{Ni}$, respectively. 


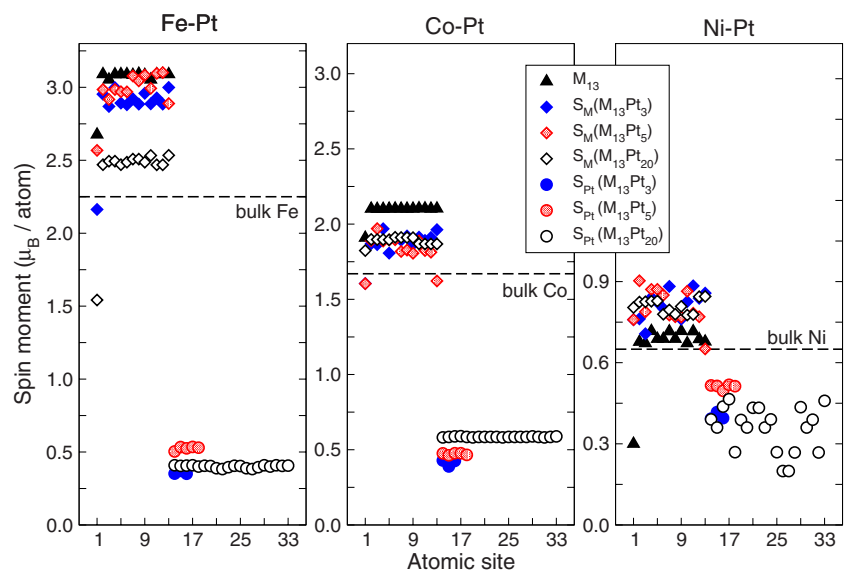

FIG. 11. (Color online) The spin moment $\left|\mathbf{S}_{i}\right|$ of each atomic site of $\mathrm{Fe}_{13} \mathrm{Pt}_{n}$ (left), $\mathrm{Co}_{13} \mathrm{Pt}_{n}$ (middle), and $\mathrm{Ni}_{13} \mathrm{Pt}_{n}$ (right) clusters. The dashed lines show the calculated bulk values of the spin moments of bcc Fe, fcc $\mathrm{Co}$, and fcc Ni, respectively. The same symbols as in Fig. 10 are used.

and Co cores, it grows with the number $n$ of $\mathrm{Pt}$ atoms but for $\mathrm{Ni}$ cores it slightly decreases with growing $n$.

In Fig. 11, the site-specific spin moments of the capped clusters are shown. Different trends are found for the core atoms: While the $\mathrm{Fe}$ and the Co spin moments are reduced by the capping, the $\mathrm{Ni}$ moments are somewhat enhanced. The Pt spin moment does not show a clear trend.

One intriguing point is the difference between the trends of Pt orbital and spin moments for the $M_{13} \mathrm{Pt}_{3} / M_{13} \mathrm{Pt}_{5}$ clusters, as $M$ varies from Fe to Ni. Here, the Pt orbital moments increase by a factor of 2 while the $\mathrm{Pt}$ spin moments are merely unchanged. A qualified explanation of this difference would require a detailed analysis of the related electronic structure which is beyond the scope of this work. We assume that the hybridization between $\mathrm{Ni}$ and $\mathrm{Pt}$ is weaker than be- tween Fe and Pt due to the different extension of the atomic orbitals. Weaker hybridization, in general, yields larger orbital moments since the hybridization mixes different $m$ states. An approximate conservation of spin moments of the $\mathrm{Pt}$ atoms while passing from $\mathrm{Fe}$ to $\mathrm{Ni}$ may be due to the fact that in each case the maximum induced spin moment on $\mathrm{Pt}$ is already reached, close to a value of $0.5 \mu_{B}$ which is comparable to the spin moment of the isoelectronic $\mathrm{Ni}$. We have checked this by additional calculations in which we placed one $\mathrm{Pt}$ atom on the top of triangular $\mathrm{Fe}_{3}\left(\mathrm{Co}_{3}, \mathrm{Ni}_{3}\right)$ clusters, which shows a similar variation of $\left|\mathbf{L}_{i}\right|$ and $\left|\mathbf{S}_{i}\right|$ as in the cluster calculations of $M_{13} \mathrm{Pt}_{3} / M_{13} \mathrm{Pt}_{5}$. In the following, a few quantitative statements are made for each group of capped clusters.

\section{A. $\mathrm{Fe}_{13} \mathrm{Pt}_{n}$ clusters}

The values of average orbital moment and average spin moment for each atomic species along with the total orbital moment $L_{\text {tot }}$ and total spin moment $S_{\text {tot }}$ for the capped clusters are defined and reported in Table VI. By adding Pt atoms to the $\mathrm{Fe}_{13}$ cluster, the $L_{\text {tot }}$ increases considerably while the $S_{\text {tot }}$ is reduced if the $\mathrm{Pt}$ overlayer is completed. This is due to a considerable reduction of the Fe spin moment in comparison with the bare $\mathrm{Fe}_{13}$ cluster. The average orbital moment $\left\langle L_{\mathrm{M}}\right\rangle$ on $\mathrm{Fe}_{13}$ decreases with increasing number of Pt atoms.

Regarding the MAE of capped $M_{13} \mathrm{Pt}_{n}$ clusters, we observe that the symmetry of the cluster determines the magnitude of the effect similar to the case in the $M_{13}$ systems. For example, we have capped four $\mathrm{Pt}$ atoms onto $\mathrm{Fe}_{13}$ with the Mackay orientation, to get the $\mathrm{Fe}_{13} \mathrm{Pt}_{4}$ cluster shown on the left panel of Fig. 12, and, after optimizing its structure, we calculated its MAE. Due to the low symmetry, the anisotropy is of second-order. It turned out to be quite large, exceeding by a factor of $10(\sim 7 \mathrm{meV} / \mathrm{cluster})$ that of the MTdistorted $\mathrm{Fe}_{13}$ and by a factor of 2 that of the JT-distorted $\mathrm{Fe}_{13}$.

TABLE VI. The orbital and spin moments for the binary Pt-M clusters in $\mu_{B} /$ atom, where we have distinguished core and shell contributions. Cluster averaged values are defined by $\left\langle L_{\mathrm{M}}\right\rangle=\frac{1}{13} \sum_{i=1}^{13}\left|\mathbf{L}_{i, \mathrm{M}}\right|$ and $\left\langle S_{\mathrm{M}}\right\rangle=\frac{1}{13} \sum_{i=1}^{13}\left|\mathbf{S}_{i, \mathrm{M}}\right|$ (M represents the 13-atom Fe, Co, and Ni clusters), $\left\langle L_{\mathrm{Pt}}\right\rangle=\frac{1}{n} \sum_{i=1}^{n}\left|\mathbf{L}_{i, \mathrm{Pt}}\right|$ and $\left\langle S_{\mathrm{Pt}}\right\rangle$ $=\frac{1}{n} \sum_{i=1}^{n}\left|\mathbf{S}_{i, \mathrm{Pt}}\right|$ (the number of Pt atoms $n=3,4,5$, and 20) $\left|\mathbf{L}_{\text {tot }}\right|$ and $\left|\mathbf{S}_{\text {tot }}\right|$ are the corresponding absolute values of the total orbital and total spin moment for every cluster.

\begin{tabular}{lcccccc}
\hline \hline Cluster & $\left\langle L_{\mathrm{M}}\right\rangle$ & $\left\langle L_{\mathrm{Pt}}\right\rangle$ & $\left\langle S_{\mathrm{M}}\right\rangle$ & $\left\langle S_{\mathrm{Pt}}\right\rangle$ & $\left|\mathbf{L}_{\text {tot }}\right|$ & $\left|\mathbf{S}_{\text {tot }}\right|$ \\
\hline $\mathrm{Fe}_{13}$ & 0.08 & & 3.05 & & 1.09 & 39.64 \\
$\mathrm{Fe}_{13} \mathrm{Pt}_{3}$ & 0.09 & 0.08 & 2.86 & 0.36 & 1.43 & 38.30 \\
$\mathrm{Fe}_{13} \mathrm{Pt}_{4}$ & 0.10 & 0.14 & 2.87 & 0.32 & 1.74 & 38.60 \\
$\mathrm{Fe}_{13} \mathrm{Pt}_{5}$ & 0.08 & 0.13 & 2.97 & 0.53 & 1.76 & 41.30 \\
$\mathrm{Fe}_{13} \mathrm{Pt}_{20}$ & 0.05 & 0.17 & 2.18 & 0.40 & 2.74 & 36.40 \\
$\mathrm{Co}_{13}$ & 0.12 & & 2.05 & & 1.52 & 26.60 \\
$\mathrm{Co}_{13} \mathrm{Pt}_{3}$ & 0.10 & 0.13 & 1.87 & 0.42 & 1.68 & 25.60 \\
$\mathrm{Co}_{13} \mathrm{Pt}_{5}$ & 0.09 & 0.16 & 1.83 & 0.47 & 2.06 & 26.10 \\
$\mathrm{Co}_{13} \mathrm{Pt}_{20}$ & 0.07 & 0.30 & 1.88 & 0.60 & 4.90 & 36.20 \\
$\mathrm{Ni}_{13}$ & 0.06 & & 0.66 & & 0.80 & 8.65 \\
$\mathrm{Ni}_{13} \mathrm{Pt}_{3}$ & 0.06 & 0.20 & 0.81 & 0.40 & 1.61 & 11.70 \\
$\mathrm{Ni}_{13} \mathrm{Pt}_{5}$ & 0.05 & 0.26 & 0.80 & 0.51 & 2.01 & 13.00 \\
$\mathrm{Ni}_{13} \mathrm{Pt}_{20}$ & 0.08 & 0.20 & 0.81 & 0.36 & 3.55 & 17.60 \\
\hline \hline
\end{tabular}



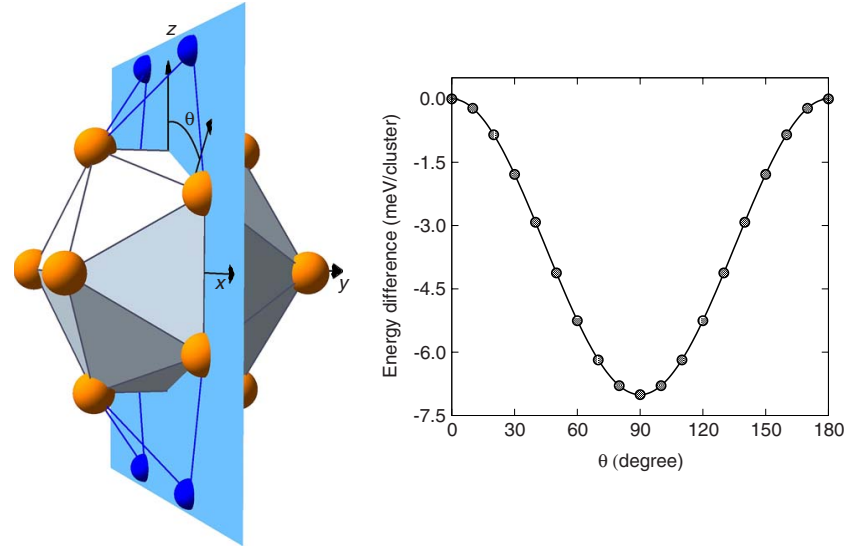

FIG. 12. (Color online) Left: the $x-z$ plane (where $\theta$ is varied) of the $\mathrm{Fe}_{13} \mathrm{Pt}_{4}$ cluster, showing the positions of the $\mathrm{Pt}$ atoms relative to the ICO in the initial unrelaxed configuration. Light (orange) and dark (blue) spheres are the $\mathrm{Fe}$ and $\mathrm{Pt}$ atoms, respectively. In the relaxed $\mathrm{Fe}_{13} \mathrm{Pt}_{4}$ cluster, two opposite edges (those adjacent to the $\mathrm{Pt}$ atoms) of the $\mathrm{Fe}_{13}$ cluster moved toward each other. Right: the $\theta$-dependent energy differences for the relaxed $\mathrm{Fe}_{13} \mathrm{Pt}_{4}$ cluster. Here, the solid curve is not a fit to the Néel formula because of the heterogeneous form of the cluster but simply a cubic spline fit to the GGA data.

\section{B. $\mathrm{Co}_{13} \mathbf{P t}_{n}$ clusters}

A ferromagnetic ground state is found to be stable for all investigated compositions of $\mathrm{Co}_{13} \mathrm{Pt}_{n}$ clusters. $L_{\text {tot }}$ increases monotonically with $n$. $\left\langle L_{\mathrm{M}}\right\rangle$ and $\left\langle L_{\mathrm{Pt}}\right\rangle$ for $\mathrm{Co}_{13} \mathrm{Pt}_{n}$ clusters show a trend similar to $\mathrm{Fe}_{13} \mathrm{Pt}_{n}$, i.e., with increasing number of Pt atoms, $\left\langle L_{\mathrm{M}}\right\rangle$ (as defined in Table VI) decreases and
$\left\langle\mathrm{L}_{\mathrm{Pt}}\right\rangle$ grows. $\left\langle\mathrm{S}_{\mathrm{M}}\right\rangle$ on $\mathrm{Co}_{13}$ is merely constant while the Ptspin moment increases monotonically with the number of $\mathrm{Pt}$ atoms on the cluster surface. This yields a high spin moment of the completely Pt-covered $\mathrm{Co}_{13}$.

\section{C. $\mathrm{Ni}_{13} \mathrm{Pt}_{n}$ clusters}

In this case, both $L_{\mathrm{tot}}$ and $S_{\text {tot }}$ show a monotonic increase with the number of Pt atoms. $\left\langle L_{\mathrm{M}}\right\rangle$ and $\left\langle L_{\mathrm{Pt}}\right\rangle$ for this cluster type do not much depend on the number of $\mathrm{Pt}$ atoms. However, a decrease in $\left\langle L_{\mathrm{Pt}}\right\rangle$ and $\left\langle S_{\mathrm{Pt}}\right\rangle$ from $\mathrm{Ni}_{13} \mathrm{Pt}_{5}$ to $\mathrm{Ni}_{13} \mathrm{Pt}_{20}$ is also observed. This is probably caused by a structural instability occurring for the latter composition upon relaxation. The geometry optimization of this cluster converges to a structure with different symmetry, where the $\mathrm{Ni}$ atoms are placed closer to the surface of the cluster as shown in Fig. 8. The reason for the segregation of $\mathrm{Ni}$ atoms toward the surface may be due to its lower surface energy compared to $\mathrm{Pt}^{72}$ Another related aspect may be observed in the right panels of Figs. 10 and 11 showing the variation in onsite orbital and spin moments. The large variations in orbital and spin moments just occur because of the structural distortion for this cluster composition. Comparing all three cases of capped clusters, we find that the presence of $\mathrm{Pt}$ atoms on $M_{13}$ affects the orientation of core orbital moments in such a way that they always prefer to be in noncollinear alignment for the $M_{13} \mathrm{Pt}_{n}$ clusters, which is not the case in the uncapped $M_{13}$ clusters. On the other hand, directions of individual spin moments remain always collinear for the same clusters indicating that they are less affected by the Pt atoms.

TABLE VII. Spin and orbital moments of the relaxed $\mathrm{Co}_{13}(\mathrm{MT})$ and $\mathrm{Ni}_{13}$ (MT) clusters, calculated with VASP and SIESTA, for an initial [001] setting of the spin direction. The $x$ and $y$ components of the spin moment are $<0.001 \mu_{B}$, hence not shown here. For the SIESTA calculations, the values of spin moments are given in terms of Mulliken populations and by direct integration of the spin density over atom-centered spheres with a radius of $1.302 \AA\left(\mathrm{Fe}_{13}, \mathrm{Co}_{13}\right)$ and $1.286 \AA\left(\mathrm{Ni}_{13}\right)$. The spin moments calculated over atom centered spheres are shown in the parentheses. The magnetic moments are given in units of $\mu_{B}$ /atom.

\begin{tabular}{|c|c|c|c|c|c|c|c|c|c|c|c|c|c|c|c|c|}
\hline \multirow[b]{3}{*}{ Atom No. } & \multicolumn{8}{|c|}{$\mathrm{Co}_{13}(\mathrm{MT})$} & \multicolumn{8}{|c|}{$\mathrm{Ni}_{13}(\mathrm{MT})$} \\
\hline & \multicolumn{4}{|c|}{ VASP } & \multicolumn{4}{|c|}{ SIESTA } & \multicolumn{4}{|c|}{ VASP } & \multicolumn{4}{|c|}{ SIESTA } \\
\hline & $L_{x}$ & $L_{y}$ & $L_{z}$ & $S_{z}$ & $L_{x}$ & $L_{y}$ & $L_{z}$ & $S_{z}$ & $L_{x}$ & $L_{y}$ & $L_{z}$ & $S_{z}$ & $L_{x}$ & $L_{y}$ & $L_{z}$ & $S_{z}$ \\
\hline 1 & 0.00 & 0.02 & 0.10 & 2.06 & 0.00 & 0.00 & 0.08 & $2.42(2.16)$ & 0.00 & -0.02 & 0.06 & 0.66 & 0.00 & 0.01 & 0.07 & $0.64(0.68)$ \\
\hline 2 & 0.00 & -0.02 & 0.10 & 2.06 & 0.00 & 0.00 & 0.08 & $2.42(2.14)$ & 0.00 & 0.02 & 0.06 & 0.66 & 0.00 & -0.01 & 0.07 & $0.64(0.68)$ \\
\hline 3 & 0.02 & 0.00 & 0.12 & 2.05 & 0.01 & 0.00 & 0.10 & $2.42(2.17)$ & 0.00 & 0.00 & 0.07 & 0.70 & -0.01 & 0.00 & 0.06 & $0.64(0.68)$ \\
\hline 4 & -0.02 & 0.00 & 0.12 & 2.05 & -0.01 & 0.00 & 0.10 & $2.42(2.14)$ & 0.00 & 0.00 & 0.07 & 0.70 & 0.01 & 0.00 & 0.06 & $0.64(0.67)$ \\
\hline 5 & 0.00 & 0.00 & 0.15 & 2.05 & 0.00 & 0.00 & 0.12 & $2.42(2.17)$ & 0.00 & 0.00 & 0.06 & 0.70 & 0.00 & 0.00 & 0.05 & $0.64(0.68)$ \\
\hline 6 & 0.00 & 0.00 & 0.15 & 2.05 & 0.00 & 0.00 & 0.12 & $2.42(2.14)$ & 0.00 & 0.00 & 0.06 & 0.70 & 0.00 & 0.00 & 0.05 & $0.64(0.67)$ \\
\hline 7 & 0.00 & 0.00 & 0.15 & 2.05 & 0.00 & 0.00 & 0.12 & $2.42(2.19)$ & 0.00 & 0.00 & 0.06 & 0.70 & 0.00 & 0.00 & 0.05 & $0.64(0.68)$ \\
\hline 8 & 0.00 & 0.00 & 0.15 & 2.05 & 0.00 & 0.00 & 0.12 & $2.42(2.16)$ & 0.00 & 0.00 & 0.06 & 0.70 & 0.00 & 0.00 & 0.05 & $0.64(0.68)$ \\
\hline 9 & -0.02 & 0.00 & 0.12 & 2.05 & -0.01 & 0.00 & 0.10 & $2.42(2.19)$ & 0.00 & 0.00 & 0.07 & 0.70 & 0.01 & 0.00 & 0.06 & $0.64(0.68)$ \\
\hline 10 & 0.02 & 0.00 & 0.12 & 2.05 & 0.01 & 0.00 & 0.10 & $2.42(2.16)$ & 0.00 & 0.00 & 0.07 & 0.70 & -0.01 & 0.00 & 0.07 & $0.64(0.68)$ \\
\hline 11 & 0.00 & 0.02 & 0.10 & 2.06 & 0.00 & 0.00 & 0.08 & $2.42(2.17)$ & 0.00 & -0.02 & 0.06 & 0.66 & 0.00 & 0.01 & 0.07 & $0.64(0.68)$ \\
\hline 12 & 0.00 & -0.02 & 0.10 & 2.06 & 0.00 & 0.00 & 0.08 & $2.42(2.19)$ & 0.00 & 0.02 & 0.06 & 0.66 & 0.00 & 0.01 & 0.07 & $0.64(0.68)$ \\
\hline 13 & 0.00 & 0.00 & 0.04 & 1.97 & 0.00 & 0.00 & 0.04 & $1.96(1.88)$ & 0.00 & 0.00 & 0.02 & 0.38 & 0.00 & 0.00 & 0.00 & $0.30(0.27)$ \\
\hline
\end{tabular}




\section{CONCLUSIONS}

The spin and orbital magnetic moments are calculated for $M_{13}$ and $M_{13} \mathrm{Pt}_{n}$ clusters using DFT methods that include spin-orbit interaction. The calculated MAEs of relaxed $M_{13}$ clusters are considerably enhanced relative to both the ideal icosahedral clusters and to the corresponding cubic bulk values. The MAEs for relaxed clusters are found to be affected in a specific way by the degree and type of relaxation. For JT-distorted clusters, we observed larger MAEs compared to those in Mackay-distorted clusters because of the lower symmetry of the former. The present calculations of $\theta$-dependent MAE can be well represented by a Néel model taking into account only the leading order of the respective symmetry. With respect to the spin and orbital moments, both the capped and the free clusters show an increased value of orbital and spin moments compared to the bulk. For the capped clusters, the spin moments on $\mathrm{Pt}$ atoms remain unaffected by the host atoms. Finally, we infer that deposited transition-metal clusters, with very large effects of relaxations, may exhibit even larger MAE values. Self-assembly of such clusters, like in case of $\mathrm{Fe}-\mathrm{Pt}$ (Ref. 13) and Co, ${ }^{15}$ may then approach the class of functional magnetic materials of use for magnetic storage devices.

\section{ACKNOWLEDGMENTS}

The authors would like to acknowledge Helmut Eschrig, Klaus Koepernik, Hubert Ebert, and Heike Herper for helpful discussions. M. E. Gruner acknowledges discussions with Karlheinz Schwarz and Peter Blaha from TU-Wien. M. Richter acknowledges discussions with Andriy Leonov from IFW Dresden. This work was supported by SFB 445 and SPP 1145. Parts of the calculations were carried out on the JUGENE supercomputer system of the John von Neumann Institute for computing at Forschungszentrum Jülich.

\section{APPENDIX: COMPARISON OF MAGNETIC MOMENTS OBTAINED FROM VASP AND SIESTA}

The magnetic moments calculated for MT-distorted $\mathrm{Co}_{13}$ and $\mathrm{Ni}_{13}$ clusters using VASP and SIESTA are depicted in Table VII. Taking a spatial summation of the spin density into account, the difference between the center and surface atoms for $\mathrm{Co}_{13}$ is calculated to be approximately $0.1 \mu_{B}$ (VASP) and $0.3 \mu_{B}$ (SIESTA), whereas it amounts to about $0.3 \mu_{B}$ (VASP) and $0.4 \mu_{B}$ (SIESTA) for $\mathrm{Ni}_{13}$. Considering the Mulliken analysis (SIESTA), this difference becomes about $0.5 \mu_{B}$ for $\mathrm{Co}_{13}$ and about $0.3 \mu_{B}$ for $\mathrm{Ni}_{13}$.

\footnotetext{
*sanjubala.sahoo@uni-due.de

${ }^{1}$ S. Polesya, O. Šipr, S. Bornemann, J. Minár, and H. Ebert, Europhys. Lett. 74, 1074 (2006).

${ }^{2}$ O. Šipr, S. Polesya, J. Minár, and H. Ebert, J. Phys.: Condens. Matter 19, 446205 (2007).

${ }^{3}$ Y. Xie and J. A. Blackman, Phys. Rev. B 66, 085410 (2002).

${ }^{4}$ O. Diéguez, M. M. G. Alemany, C. Rey, P. Ordejón, and L. J. Gallego, Phys. Rev. B 63, 205407 (2001).

${ }^{5}$ A. V. Postnikov, P. Entel, and J. M. Soler, Eur. Phys. J. D 25, 261 (2003)

${ }^{6}$ H. M. Duan and Q. Q. Zheng, Phys. Lett. A 280, 333 (2001).

${ }^{7}$ S. E. Apsel, J. W. Emmert, J. Deng, and L. A. Bloomfield, Phys. Rev. Lett. 76, 1441 (1996).

${ }^{8}$ B. J. Winter, T. D. Klots, E. K. Parks, and S. J. Riley, Z. Phys. D 19, 375 (1991); J.-O. Bovin, and J.-O. Malm, ibid. 19, 293 (1991).

${ }^{9}$ B. I. Dunlap, Phys. Rev. A 41, 5691 (1990).

${ }^{10}$ M. L. Tiago, Y. Zhou, M. M. G. Alemany, Y. Saad, and J. R. Chelikowsky, Phys. Rev. Lett. 97, 147201 (2006).

${ }^{11}$ P. Entel, M. E. Gruner, G. Rollmann, A. Hucht, S. Sahoo, A. T. Zayak, H. C. Herper, and A. Dannenberg, Philos. Mag. 88, 2725 (2008).

${ }^{12}$ G. Rollmann, M. E. Gruner, A. Hucht, R. Meyer, P. Entel, M. L. Tiago, and J. R. Chelikowsky, Phys. Rev. Lett. 99, 083402 (2007); M. E. Gruner and P. Entel, J. Phys.: Condens. Matter 21, 293201 (2009).

${ }^{13}$ M. E. Gruner, G. Rollmann, P. Entel, and M. Farle, Phys. Rev. Lett. 100, 087203 (2008).

${ }^{14}$ T. Futschek, J. Hafner, and M. Marsman, J. Phys.: Condens. Matter 18, 9703 (2006).
}

${ }^{15}$ R. Xiao, D. Fritsch, M. D. Kuz'min, K. Koepernik, H. Eschrig, M. Richter, K. Vietze, and G. Seifert, Phys. Rev. Lett. 103, 187201 (2009)

${ }^{16}$ L. M. Sandratskii, Adv. Phys. 47, 91 (1998).

${ }^{17}$ L. M. Sandratskii and J. Kübler, Mod. Phys. Lett. B 10, 189 (1996).

${ }^{18}$ T. Oda, A. Pasquarello, and R. Car, Phys. Rev. Lett. 80, 3622 (1998).

${ }^{19}$ D. Hobbs, G. Kresse, and J. Hafner, Phys. Rev. B 62, 11556 (2000).

${ }^{20}$ S. Lounis, P. Mavropoulos, R. Zeller, P. H. Dederichs, and S. Blügel, Phys. Rev. B 75, 174436 (2007).

${ }^{21}$ T. O. Strandberg, C. M. Canali, and A. H. MacDonald, Nature Mater. 6, 648 (2007).

${ }^{22}$ P. Gambardella, S. Rusponi, M. Veronese, S. S. Dhesi, C. Grazioli, A. Dallmeyer, I. Cabria, R. Zeller, P. H. Dederichs, K. Kern, C. Carbone, and H. Brune, Science 300, 1130 (2003).

${ }^{23}$ F. Luis, J. Bartolomé, F. Bartolomé, M. J. Martínez, L. M. García, F. Petroff, C. Deranlot, F. Wilhelm, and A. Rogalev, J. Appl. Phys. 99, 08 G705 (2006).

${ }^{24}$ J. Bansmann, S. H. Baker, C. Binns, J. A. Blackman, J.-P. Bucher, J. Dorantes-Dávila, V. Dupuis, L. Favre, D. Kechrakos, A. Kleibert, K.-H. Meiwes-Broer, G. M. Pastor, A. Perez, O. Toulemonde, K. N. Trohidou, J. Tuaillon, and Y. Xie, Surf. Sci. Rep. 56, 189 (2005).

${ }^{25}$ S. Dennler, J. Morillo, and G. M. Pastor, J. Phys.: Condens. Matter 16, S2263 (2004).

${ }^{26}$ M. Muñoz-Navia, J. Dorantes-Dávila, and G. M. Pastor, J. Phys.: Condens. Matter 16, S2251 (2004).

${ }^{27}$ M. Tsujikawa, A. Hosokawa, and T. Oda, Phys. Rev. B 77, 
054413 (2008).

${ }^{28}$ R. Félix-Medina, J. Dorantes-Dávila, and G. M. Pastor, Phys. Rev. B 67, 094430 (2003).

${ }^{29}$ B. Nonas, I. Cabria, R. Zeller, P. H. Dederichs, T. Huhne, and H. Ebert, Phys. Rev. Lett. 86, 2146 (2001).

${ }^{30}$ A. N. Andriotis and M. Menon, Phys. Rev. Lett. 93, 026402 (2004).

${ }^{31}$ S. Rohart, C. Raufast, L. Favre, E. Bernstein, E. Bonet, and V. Dupuis, Phys. Rev. B 74, 104408 (2006).

${ }^{32}$ Š. Pick, V. S. Stepanyuk, A. L. Klavsyuk, L. Niebergall, W. Hergert, J. Kirschner, and P. Bruno, Phys. Rev. B 70, 224419 (2004).

${ }^{33}$ G. M. Pastor, J. Dorantes-Dávila, S. Pick, and H. Dreyssé, Phys. Rev. Lett. 75, 326 (1995).

${ }^{34}$ L. Fernández-Seivane and J. Ferrer, Phys. Rev. Lett. 99, 183401 (2007).

${ }^{35}$ Ch. Köhler, Th. Frauenheim, B. Hourahine, G. Seifert, and M. Sternberg, J. Phys. Chem. A 111, 5622 (2007).

${ }^{36}$ J. Hong and R. Q. Wu, J. Appl. Phys. 93, 8764 (2003).

${ }^{37}$ D. Fritsch, K. Koepernik, M. Richter, and H. Eschrig, J. Comput. Chem. 29, 2210 (2008).

${ }^{38}$ P. Błoński and J. Hafner, Phys. Rev. B 79, 224418 (2009).

${ }^{39}$ R. Singh and P. Kroll, Phys. Rev. B 78, 245404 (2008).

${ }^{40}$ F. Aguilera-Granja and A. Vega, Phys. Rev. B 79, 144423 (2009).

${ }^{41}$ J. Lyubina, I. Opahle, K.-H. Müller, O. Gutfleisch, M. Richter, M. Wolf, and L. Schultz, J. Phys.: Condens. Matter 17, 4157 (2005).

${ }^{42}$ G. Kresse and J. Furthmüller, Comput. Mater. Sci. 6, 15 (1996).

${ }^{43}$ G. Kresse and J. Furthmüller, Phys. Rev. B 54, 11169 (1996).

${ }^{44}$ G. Kresse and D. Joubert, Phys. Rev. B 59, 1758 (1999).

${ }^{45}$ J. M. Soler, E. Artacho, J. D. Gale, A. Gracía, J. Junquera, P. Ordejón, and D. Sánchez-Portal, J. Phys.: Condens. Matter 14, 2745 (2002).

${ }^{46}$ K. Koepernik and H. Eschrig, Phys. Rev. B 59, 1743 (1999).

${ }^{47}$ J. P. Perdew, in Electronic Structure of Solids'91, edited by P. Ziesche and H. Eschrig (Akademie Verlag, Berlin, 1991), pp. 11-20.

${ }^{48}$ P. E. Blöchl, Phys. Rev. B 50, 17953 (1994).

${ }^{49}$ G. H. O. Daalderop, P. J. Kelly, and M. F. H. Schuurmans, Phys. Rev. B 41, 11919 (1990).

${ }^{50}$ J. Junquera, Ó. Paz, D. Sánchez-Portal, and E. Artacho, Phys.
Rev. B 64, 235111 (2001).

${ }^{51}$ N. Troullier and J. L. Martins, Phys. Rev. B 43, 1993 (1991).

${ }^{52}$ J. P. Perdew, K. Burke, and M. Ernzerhof, Phys. Rev. Lett. 77, 3865 (1996).

${ }^{53}$ L. Fernández-Seivane, M. A. Oliveira, S. Sanvito, and J. Ferrer, J. Phys.: Condens. Matter 18, 7999 (2006).

${ }^{54}$ http://www.fplo.de/

${ }^{55}$ J. P. Perdew and Y. Wang, Phys. Rev. B 45, 13244 (1992).

${ }^{56}$ H. Eschrig, M. Richter, and I. Opahle, in Relativistic Solid State Calculations. Relativistic Electronic Structure Theory-Part II. Applications, edited by P. Schwerdtfeger (Elsevier, Amsterdam, 2004), pp. 723-776.

${ }^{57}$ Q.-M. Ma, Z. Xie, J. Wang, Y. Liu, and Y.-C. Li, Phys. Lett. A 358, 289 (2006).

${ }^{58}$ G. Rollmann, S. Sahoo, and P. Entel, Phys. Status Solidi A 201, 3263 (2004).

${ }^{59}$ G. Rollmann, P. Entel, and S. Sahoo, Comput. Mater. Sci. 35, 275 (2006).

${ }^{60}$ G. Rollmann, Ph.D. thesis, University of Duisburg-Essen, 2007.

${ }^{61}$ H. A. Jahn and E. Teller, Proc. R. Soc. London 161, 220 (1937).

${ }^{62}$ A. L. Mackay, Acta Crystallogr. 15, 916 (1962).

${ }^{63}$ P. Entel, M. E. Gruner, A. Hucht, R. Meyer, G. Rollmann, S. Sahoo, and S. K. Nayak, in Mesoscopic, Nanoscopic and Macroscopic Materials, edited by S. M. Bose, S. N. Behera, and B. K. Roul, AIP Conf. Proc. No. 1063, (AIP, New York, 2008), pp. 3-17.

${ }^{64}$ Q.-M. Ma, Z. Xie, J. Wang, Y. Liu, and Y.-C. Li, Solid State Commun. 142, 114 (2007)

${ }^{65}$ Z. Xie, Q.-M. Ma, Y. Liu, and Y.-C. Li, Phys. Lett. A 342, 459 (2005).

${ }^{66}$ R. A. Guirado-López, J. Dorantes-Dávila, and G. M. Pastor, Phys. Rev. Lett. 90, 226402 (2003).

${ }^{67}$ M. E. Gruner, P. Entel, I. Opahle, and M. Richter, J. Mater. Sci. 43, 3825 (2008).

${ }^{68}$ L. Néel, C. R. Acad. Sci., Ser. I: Math. 237, 1468 (1953).

${ }^{69}$ L. Néel, J. Phys. Radium 15, 225 (1954)

${ }^{70}$ S. V. Halilov, A. Ya. Perlov, P. M. Oppeneer, A. N. Yaresko, and V. N. Antonov, Phys. Rev. B 57, 9557 (1998).

${ }^{71}$ H. Ebert, S. Bornemann, J. Minár, P. H. Dederichs, R. Zeller, and I. Cabria, Comput. Mater. Sci. 35, 279 (2006).

${ }^{72}$ J. A. Alonso and N. H. March, Electrons in Metals and Alloys (Academic, London, 1989), pp. 433-444. 